\title{
The effects of mirror reflections and planar rotations of pictures on the shape percept of the depicted object
}

\author{
Els V K Cornelis $\uparrow$, Andrea $\mathrm{J}$ van Doorn, Johan Wagemans $\S$ \\ Industrial Design, Delft University of Technology, Landbergstraat 15, 2628 CC Delft, The Netherlands; \\ e-mail: els.cornelis@psy.kuleuven.be; - also Laboratory of Experimental Psychology, University of \\ Leuven, Tiensestraat 102, B 3000 Leuven, Belgium; § University of Leuven \\ Received 5 June 2008, in revised form 4 July 2008; published online 15 September 2009
}

\begin{abstract}
Mirror reflections and planar rotations of a picture do not result in any variations concerning the internal geometrical layout of the objects depicted in the picture. We examined to what extent these picture plane transformations gave rise to perceptual differences. A large set of pictures was generated by mirror-reflecting and rotating a set of six original photographs in the picture plane. We externalised the percepts of the depicted objects by using a direct perceptual method: the gauge-figure method. Participants had to adjust a gauge figure so that it seemed to be painted on the surface of the depicted object. From an extensive set of settings collected this way, we computed for each picture the three-dimensional interpretation-or pictorial relief - of the depicted object. On the basis of this set of pictorial reliefs, we addressed the effects of mirror reflections and rotations of pictures on the shape percept of the depicted object. Mirror-reflecting a picture around the horizontal axis resulted in large differences in pictorial reliefs, whereas mirror-reflecting pictures around the vertical axis resulted in only small differences in pictorial reliefs. Clockwise $90^{\circ}, 180^{\circ}$, and $270^{\circ}$ rotation affected the pictorial relief significantly. In all cases, the differences between the pictorial reliefs could be resolved by affine transformations, and could thus be ascribed to different solutions of the depth ambiguities inherent in pictures.
\end{abstract}

\section{Introduction}

\subsection{On looking at and into pictures}

A picture is a special kind of object: it has a dual identity. On the one hand, it has a physical existence: when printed, it is a piece of paper covered with pigment blobs that are spatially organised in some way. On the other hand, it goes beyond this sheer physicality by representing what is depicted. One can observe both aspects. When looking at the picture surface one just considers the flat piece of paper; looking into the picture one observes a depicted scene (Deręgowski 1989; Gibson 1979; Koenderink and van Doorn 2003; Pirenne 1970). However, a two-dimensional (2-D) picture does not fully specify the actual layout of the depicted three-dimensional (3-D) object or scene. In fact, an infinite number of 3-D scenes or objects can produce one and the same picture. For instance, various 3-D objects, similar but differing by depth scaling and/or shearing, may generate the same picture (Belhumeur et al 1999). The projection of a 3-D space onto a 2-D surface causes a lack of information concerning the depth dimension, which makes every picture inherently ambiguous. Therefore, when looking into a picture, there are in principle numerous possible interpretations of the depiction.

In the case of a photograph, a depiction is the result of a registration made by a camera from a specific viewpoint and with a lens with a specific focal length. This vantage point defines the correct viewpoint for looking into the picture. A correct viewpoint concerns not only the right viewing direction relative to the picture plane, but also the right distance between the observer and the picture (ie the focal length of the lens of the camera multiplied by the degree of enlargement of the photograph). Under constrained conditions, looking into the picture from this specific viewpoint produces an isomorphism between the actual array and the pictorial array (Gibson 1954). When changing one's actual viewpoint in relation to a stationary picture, the depicted 
objects do not change; the spatial organisation of the depiction evidently stays the same. However, the retinal image of the depicted scene varies when looking at the picture from different viewpoints: geometrical deformations take place. For instance, if one takes the correct viewpoint when looking at the painting on the ceiling of the Church of St Ignatius in Rome (indicated by a star in the centre of the nave), the depth impression is compelling. When deviating from this correct viewpoint, deformations in the depicted scene become obvious. However, it seems that people's depth impression when looking into a picture is generally rather robust against taking incorrect viewpoints (eg Cutting 1988; Kubovy 1986; Pirenne 1970).

Besides the position of the observer, it is also possible to vary the orientation of the picture itself. When keeping the position and thus the viewpoint of the observer constant, but changing the orientation of a picture by rotating it within the picture plane, the layout of the projection onto the retina is different compared with the non-rotated version. There are, however, no geometrical deformations: the internal geometrical layout of the projection onto the retina does not alter. In the present research, we investigated the perceptual effects of looking into pictures that have been rotated and mirror-reflected within the picture plane.

\subsection{On orientation of pictures and figures}

When altering the orientation of a picture by applying picture plane rotations and/or mirror reflections, the internal geometrical layout of the projection onto the retina does not change. Therefore, one might expect that this kind of isomorphic transformation would have no effect on the perception of a depicted object. A striking example of the opposite, however, was observed by Mach (1886/1959) for the case of a simple, regular, flat figure: a square rotated about an angle of $45^{\circ}$ does not look like a square any more but as a diamond. In this case, the perceptual identity of the figure changes by altering its orientation. Furthermore, Mach stated that the effect of orientation on the apparent shape manifests itself differently depending on the degree of rotation of the figure with respect to a specific reference orientation (here, he used a simple, irregular, flat figure). He pointed out that the "affinity of form is apparent" (page 107) for images mirror-reflected around the vertical axis as well as for images rotated $180^{\circ}$ in relation to their original version. In contrast, the affinity of form would not be easily perceptible between mirror images of a figure around the horizontal axis and the original version. Somewhat later, Dearborn (1899, as cited in Rock 1973) studied the effect of orientation of figures on their phenomenal appearance by asking participants if they recognised figures, previously shown in an upright view in the learning phase, in various orientations in the test phase. Recognition performance varied from best to poor for, respectively, the $0^{\circ}$-rotated version, the left-right mirrored version, the $180^{\circ}$-rotated version, the up-down mirrored version, the $90^{\circ}$-rotated version, and the $270^{\circ}$-rotated version. Since then, supporting evidence for the effect of orientation on object recognition has been repeatedly demonstrated. For instance, naming performance was found to deteriorate with increasing angular deviation from the upright orientation (reaction time: Jolicoeur 1985; accuracy: Lawson and Jolicoeur 1998; for a review see Lawson 1999).

Although changing the orientation of a figure does not result in any variations in the internal geometrical layout of the projection onto the retina, the studies cited above have nevertheless found supporting evidence for the effect of orientation of (generally 2-D) figures or line drawings on performance. From these types of studies, however, it is difficult to find out what component provided the major contribution to the reported results: the more basic perceptual component or the more cognitive recognition component. In other words, are the reported results to a greater or to a lesser degree related to the perceptual effects of the manipulation (do the depicted 
objects, oriented in different ways, also look different? Are they perceived differently?) or to the memory representations privileging specific orientations?

In the present and earlier (Cornelis et al 2003) research, we focused on the more basic, perceptual component. We used an entirely different approach to investigate the perceptual shape equivalence between pictures presented in various orientations compared with the studies mentioned above. The participants were not asked to perceptually compare objects or figures shown in various orientations, nor were they asked to recognise or name the objects presented; they only performed a simple visual task (as described in Koenderink et al 1992). Participants were presented with a 2-D picture of a 3-D object on a monitor and were asked to adjust a thumbtack-like figure - the gauge figure - that was superimposed on the surface of the object until it seemed to visually fit the local surface. The participant's setting of the gauge figure revealed the perceived orientation of the local tangent plane of the surface. On the basis of an extensive set of gauge-figure settings, performed sequentially in random order at many different positions on the surface, the 3-D interpretations-or pictorial reliefs - of the depicted objects were computed. Externalising the percepts by this method made it possible to investigate the effect of transformations applied to the picture on the 3-D interpretation of the depicted object in a more direct way, ie by comparing the pictorial reliefs based on pictures differing by the specific transformation.

In Cornelis et al (2003) we investigated the effect of mirror reflection around the vertical and the horizontal axis on the shape percept of the depicted object. Hereto, the pictorial reliefs based on the original photographs were compared with those of the mirror-reflected counterparts. We found that the pictorial reliefs of the up-down mirror-reflected pictures were quite different from the pictorial reliefs based on the original counterparts; the pictorial relief differences between the left-right mirrorreflected versions and the original photographs were minimal. Both of these findings confirmed Mach's intuitions about affinity of form as discussed before. For both cases, the differences between the transformed versions and the originals could be described in a very specific way; they were causally defined by different solutions of the ambiguous visual information inherent in the presented pictures and could be ascribed to depth scaling and, especially, shears. Presenting pictures in different orientations by mirror-reflecting them thus apparently triggered other equally valid interpretations as when presenting the picture in its original orientation.

\subsection{The present research}

The present research is an extension of the previous study described above (Cornelis et al 2003) in two ways.

First, the set of picture plane transformations studied has been broadened, containing not only mirror reflections but also $90^{\circ}, 180^{\circ}$, and $270^{\circ}$ rotations. This way we obtain a more elaborate overview of the effects of picture-plane transformations not affecting the size or the position of the picture on shape perception - ie the full set of Euclidean isometries excluding translations. Mirror reflections and rotations are both picture-plane transformations that maintain relative distances and the position of the centre of the picture. However, mirror reflections of objects probably happen less frequently in the everyday world than do rotations of objects. We might therefore wonder how our visual system is attuned to both kinds of picture-plane transformations. Furthermore, in studies focusing on mental rotation, starting with Shepard and Metzler (1971), and generally reporting that the greater the angle of rotation (in the picture plane or in depth) between the objects of a pair, the longer it takes to decide if the two objects are identical, both mirror reflections and rotations were used in a rather specific way. Mirror-reflected objects were to be considered as 'different' objects; rotated objects were to be considered as 'identical' objects. It is therefore of particular importance 
to find out more about the specific effects of rotations and mirror-reflections on the shape perception of (depicted) objects.

Second, in the studies cited above, the effect of orientation was studied by presenting pictures or figures in the 'right' (upright, previously learned, or canonical orientation) and in other, 'disoriented', views, be it by rotating or mirror-reflecting them. In Cornelis et al (2003), the effect of mirror reflections on the shape percept was studied by comparing the pictorial reliefs of the original photographs, showing the torso in the usual, upright position, with the pictorial reliefs of the mirror-reflected versions. In the present study we do not specifically focus on the comparison between shape percepts based on the original view and the disoriented view, as has been done in the previous study. Here, the effect of picture-plane transformations on the shape percept of the depicted object is examined more extensively by performing not only the comparisons between the pictorial reliefs of the original pictures and their manipulated versions but by also performing every possible comparison between the pictorial reliefs of pictures differing by the specific transformation. This present approach overcomes a few difficulties encountered in the 2003 study. In the 2003 study, other variables besides the transformation might have been of importance in explaining the observed effects. For example, since the depicted object was a torso lit from top left in the original photograph, the large effect of the up-down mirror reflection could possibly be ascribed to the unnatural and unusual view of the torso. The present approach, including every possible comparison between the pictorial reliefs of pictures differing by the specific transformation, allows us to gain more insight into the importance of these kinds of possible contributing variables on the shape percept of the depicted object, presented in pictures in various orientations. For instance, by including horizontally oriented pictures as well, we can test whether the effect of lighting direction is the same or not, and, by including many different orientations away from vertical, we can test whether the similarities of the percepts follow the similarities of the views.

In the remainder of this article, we first report the study of the effects of mirror reflection (i) around the long axis of the picture frame; (ii) around both the long and the short axes of the picture frame (equivalent to a $180^{\circ}$ rotation); (iii) around the short axis of the picture frame, on the shape percept of the depicted object. In the second part of this article we focus on the effect of clockwise rotation of a picture in the picture plane by (i) $90^{\circ}$; (ii) $180^{\circ}$; and (iii) $270^{\circ}$ on shape perception.

\section{General method}

\subsection{Stimuli}

A plastic, female shop-window mannequin showing diffuse reflection and lit from top left was photographed in gray-scale with a Nikon Coolpix 990 digital camera. We chose this specific object since it has a smooth surface and a generic geometrical structure.

A set of six original photographs was prepared: three of a frontal view (indicated with $060^{\circ}, 090^{\circ}$, and $120^{\circ}$ ) and three of a dorsal view (indicated with $220^{\circ}, 250^{\circ}$, and $280^{\circ}$ ) shown in figure 1 . The different poses were obtained by rotating the torso around its vertical axis between the shots. The angle of rotation was set at $30^{\circ}$, both for the frontal views and for the dorsal views. This pose difference was chosen in order to bring sufficient variation in the stimulus material while maintaining sufficient overlap between the poses. The pose difference was the only variation between photographs: position of the torso, camera, and light source remained constant.

Transformations in the picture plane were applied to all six original photographs. These photographs were rotated $90^{\circ}, 180^{\circ}$, and $270^{\circ}$. In addition, the original photographs were left-right mirror-reflected, and these mirror-reflected versions were also rotated $90^{\circ}, 180^{\circ}$, and $270^{\circ}$ (figure 2 ). In total, we thus obtained a stimulus set of 48 pictures [6 poses $\times(7$ transformations +1 original photograph $)$ ]. 

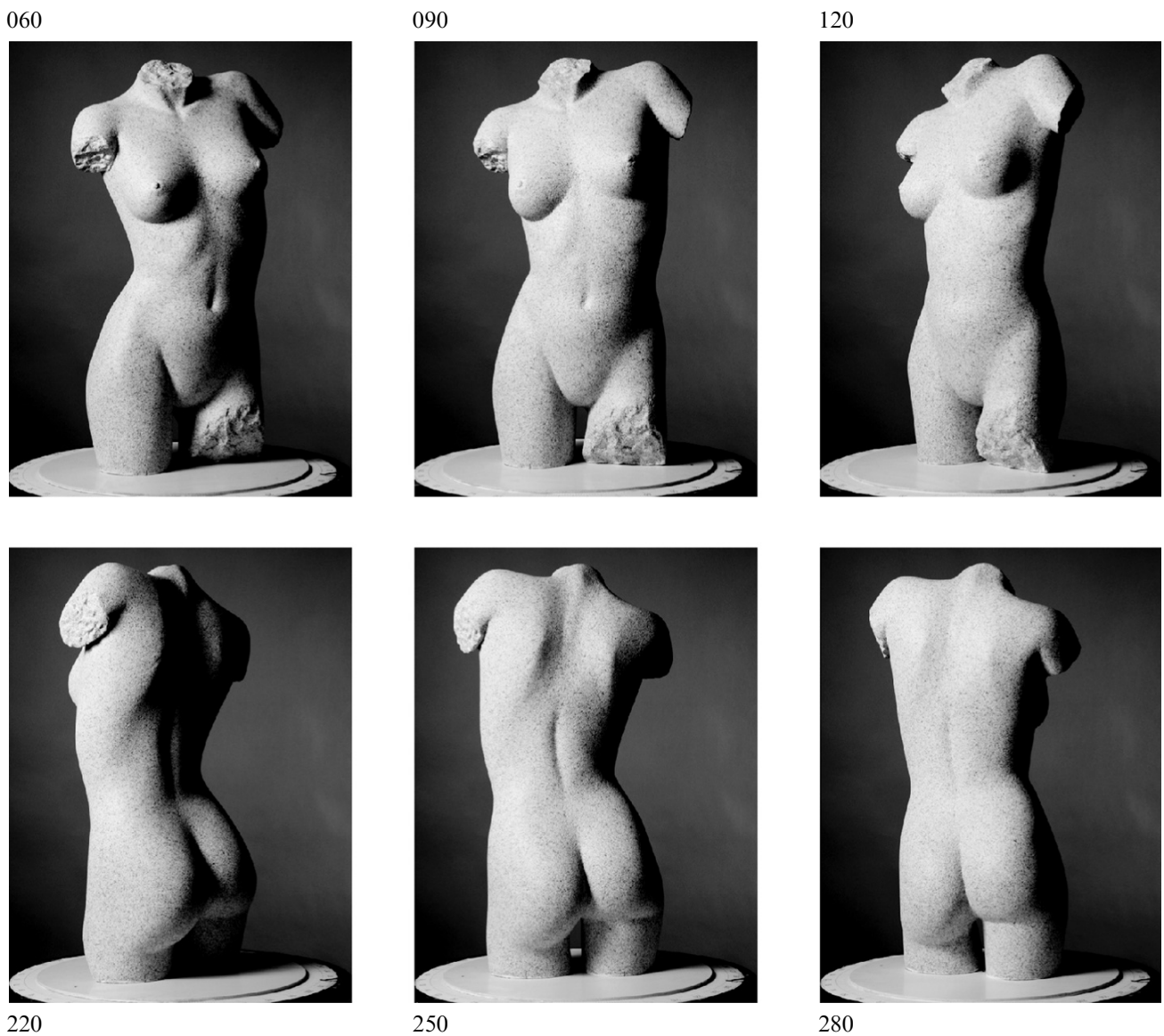

220

250

280

Figure 1. Photographs of the same object-a female shop-window mannequin-photographed in six different poses. The set of photographs contained three frontal views (poses 060, 090, and 120 ) and three dorsal views (poses 220, 250, and 280), each differing by $30^{\circ}$.

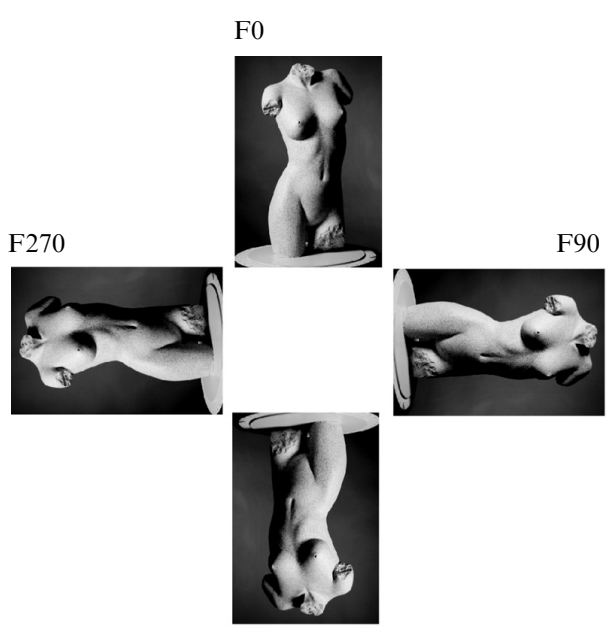

(a)

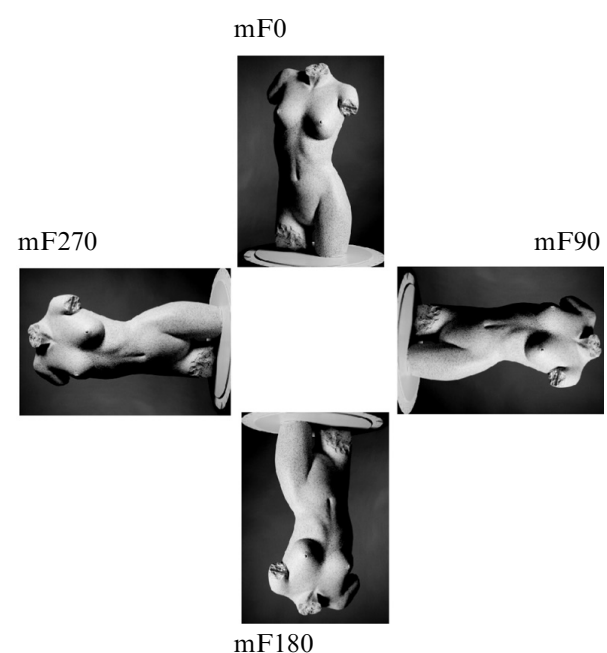

(b)

Figure 2. Total set of transformations based on one of the six original photographs (pose 060). The original picture (a) and its mirror-reflected version (b) were both rotated by $90^{\circ}, 180^{\circ}$, and $270^{\circ}$. F, fiducial; $\mathrm{m}$, mirror-reflected around the long axis of the picture; 0 , not rotated; 90 , rotated by $90^{\circ} ; 180$, rotated by $180^{\circ} ; 270$, rotated by $270^{\circ}$. 


\subsection{Procedure}

The participants were asked to adjust a gauge figure, superimposed on the depicted torso, so that it looked as if fitting in the scene (also see Koenderink et al 1992). The gauge figure was similar to a thumbtack: it consisted of the outline of an ellipse with a line segment sticking out from the centre of the ellipse. The long axis of the ellipse was kept constant at 40 pixels; the maximum length of the line segment measured 20 pixels. By manipulating a trackball, the shape and the orientation of the gauge figure could be adjusted. When adjusted appropriately, the gauge figure had to look like a circular figure as painted onto the torso's surface, with a line segment sticking out in the normal direction (figure 3). Then, the setting of the gauge figure reflected the perceived attitude of the local surface patch. When the participant perceived the gauge figure locally fitting the surface, he/she clicked a button on the trackball. The attitude parameters (slant and tilt value) as indicated by the gauge figure setting, were saved. Next, the gauge figure appeared at another location on the surface of the torso.

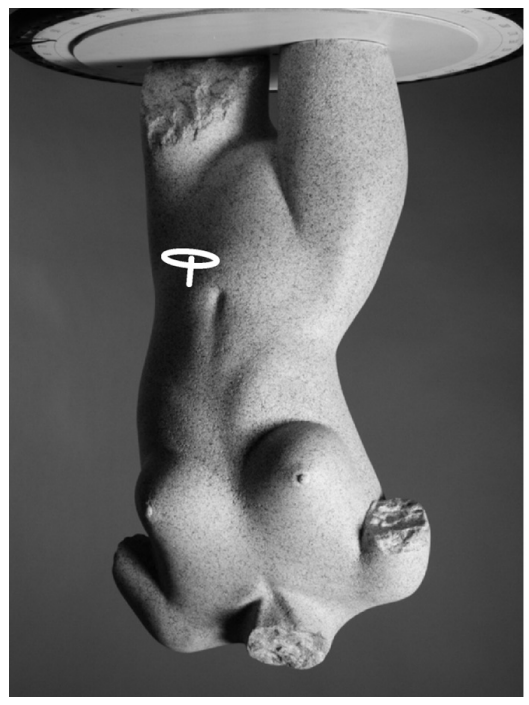

(a)

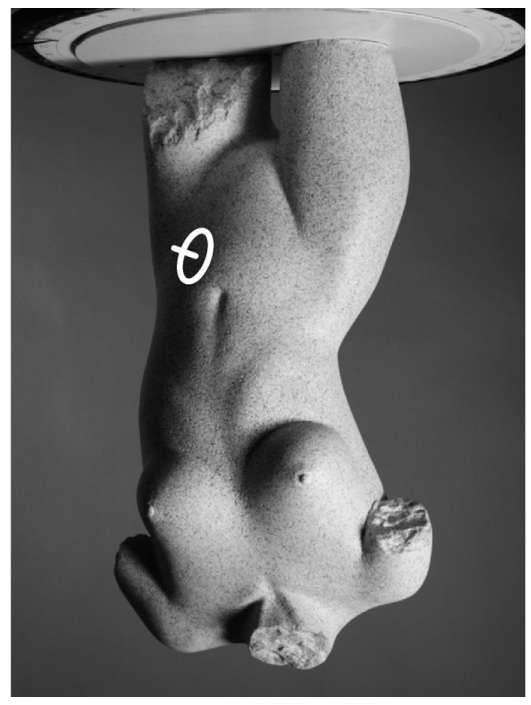

(b)

Figure 3. A gauge figure was superimposed on the torso's surface. Participants were asked to adjust the shape and the orientation of the gauge figure until it seemed to be a circle painted on the object's surface. On the left (a) an example of the figure not fitting the local surface of the depicted torso. On the right (b) an example of the figure that perceptually seemed to fit the local surface. In the experiment, the actual size of the gauge figure was smaller than is shown here; furthermore, its colour was red.

The location of the gauge figure was selected randomly out of a pool of locations, one at a time. The pool of locations of the gauge figure was determined by the barycentra specified by a triangulation. Each picture was triangulated in the following way. First, the contour of the object was traced on the image plane. Subsequently, a grid of equilateral triangles was superimposed on top of the image plane within the traced contour of the depicted object. Participants were not aware of the triangulation when performing the task.

One session probed 483 to 528 locations on the torso-the number of locations depending on the pose in which the torso was photographed. For instance, one session with the use of the picture, pose 120, measured 526 locations on the torso and thus, for this specific picture, 526 response settings were collected in one session. Each participant ran three sessions per picture. Sessions per picture did not differ from each other; measurements were thus repeated, except for the order of the locations where the gauge figure appeared. All measurements were performed randomly over picture transformations. 
The picture was displayed in the centre of a 21-inch flat computer screen (LACIE electron 22 blue). The size of the picture was $600 \times 800$ screen pixels. The resolution of the screen was $1024 \times 1280$ pixels. Viewing conditions were as follows. The monitor was placed in a frontoparallel position relative to the participant. The centre of the picture was straight-ahead with respect to the viewpoint of the participant. The eye-to-screen distance was $50 \mathrm{~cm}$. Head movements were restricted to a minimum by using a chin-rest. The room was somewhat darkened but the contours of the screen were still dimly visible. Viewing was monocular since previous research (Koenderink et al 1994) has shown that, compared to binocular viewing, monocular viewing increases the depth impression in pictures.

\subsection{Participants}

The four participants (AD, EC, HR, and $\mathrm{KJ}$ ) had normal or corrected-to-normal vision. KJ did not have any experience with psychophysical experiments. HR and KJ had not seen the actual object at the time of the experiment. [Note: The data of AD, $\mathrm{EC}$, and HR based on the original photographs and the left-right and up-down mirror-reflected versions were previously used in Cornelis et al (2003).]

\subsection{General data evaluation}

Altering the shape and the orientation of the elliptical gauge figure until it visually fitted the object's surface patch (figure 3) felt natural to all participants. One session, consisting of setting the gauge figure 483 to 528 times, took somewhat less than half an hour to complete. Local attitude settings were gathered for all pictures from AD and EC; HR and KJ did only part of the stimulus set (HR: poses 060 and 280; $\mathrm{KJ}$ : poses 060, 090, and 120). All participants ran three sessions per picture.

The setting of the shape and the orientation of the gauge figure indicated the perceived local attitude of the surface at the position of the probe. On the basis of the shape and the orientation of the ellipse, two local attitude parameters, slant and tilt of the surface patch, were extracted as described in Koenderink et al (1992; see also figure 4).

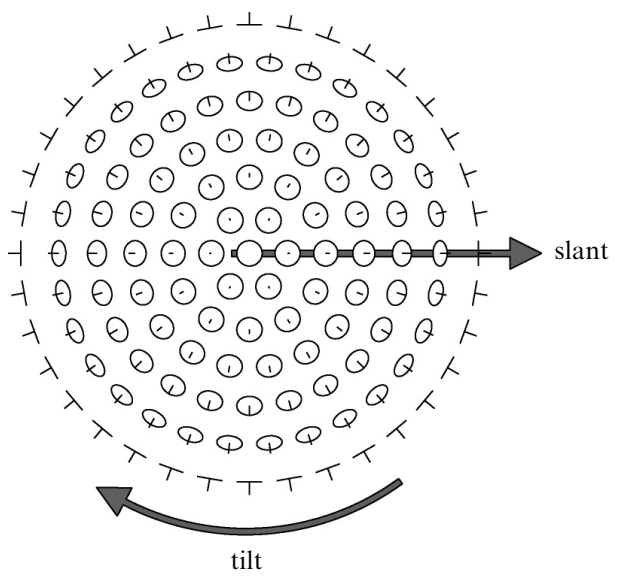

Figure 4. A set of possible gauge figure settings. The settings of the gauge figure define the two local attitude parameters - slant and tilt. The shape of the ellipse reflects the slant; the orientation of the ellipse indicates the tilt. The slant is measured as the angle between the line of sight and the local surface normal. The tilt is the direction of the slant.

On the basis of the slant and tilt values collected over the entire area of the depicted object in one session, depth gradients were calculated at the position of each probe. Next, depth differences over the faces of the triangulation were computed. The mean of the depth values was set to zero, so as to eliminate a depth offset of the pictorial relief. Subsequently, depth values were obtained by using the least squares method to achieve the best fit with the collected attitude settings. Finally, the depth values based on attitude settings of each session were averaged over the three sessions per picture. The complete data set of $\mathrm{AD}$ and $\mathrm{EC}$ contained a pictorial relief for each picture; the data set of $\mathrm{HR}$ and $\mathrm{KJ}$ consisted of pictorial reliefs for a subset of the complete picture set. 
An example of a profile view of a pictorial relief (ie obtained from the $180^{\circ}$-rotated versions of the original photograph pose 060 for $\mathrm{KJ}$ ) is shown in figure 5 .

In the remainder of this article, we are first addressing the effect of mirror reflection of a picture on the shape percept of the depicted object; second, we are focusing on the effect of rotation of a picture on shape perception. We examined the effects of both transformations using the same picture and data set as described in the general method section, but performing different comparisons between the pictorial reliefs.

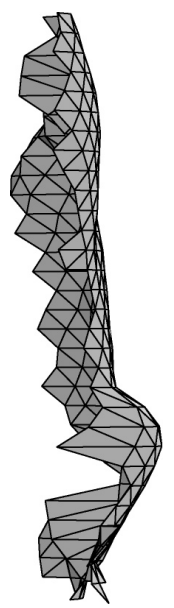

Figure 5. Profile view of the pictorial relief of the depicted object obtained from the $180^{\circ}$-rotated version (F180) of the original photograph pose 060 of participant $\mathrm{KJ}$.

3 The effect of mirror reflection of pictures on the shape percept of the depicted object The mirror reflections studied consisted of (i) mirror reflection around the long axis of the picture frame; (ii) $180^{\circ}$ rotation; and (iii) mirror reflection around the short axis of the picture frame (figure 6). We included the $180^{\circ}$ rotation because it can be considered as a combination of a mirror reflection around the long axis and around the short axis of the picture frame. By comparing the effect of a $180^{\circ}$ rotation with the separate effects of both mirror reflections, it is possible to reveal possible interaction effects of the two mirror-reflection components.

In order to gain insight into the effects of the three transformations applied, the total set of pictures as described in section 2 was organised in a specific way. First, a set of reference pictures was selected from the total set of pictures. Subsequently, we collected three comparison pictures per reference picture. The comparison pictures were transformed versions of the reference pictures and were categorised according to the mirror reflection applied. Comparisons were performed between the pictorial reliefs based on the reference picture and their transformed versions.

Furthermore, in order to investigate if the transformations would have the same influence on the shape percept in different picture-frame orientations, the pictureswhich all had a rectangular format - were divided into two sets of orientation: a vertical one and a horizontal one. In the vertically oriented picture set, the long axis of the picture frame (and thus the depicted torso) was oriented vertically; in the horizontally oriented picture set, the long axis of the picture frame (and the depicted torso) was oriented horizontally. Both orientation sets were organised according to the same types of transformations.

\subsection{Organisation of stimuli}

The entire picture set was split up into four groups. An example of a complete grouping scheme (on the basis of one of the six original photographs, pose 060) is presented 
in figure 6. The pictures in the left column form the reference set. The other three sets, the comparison sets, are to be considered in relation to the pictures of the reference set. The first comparison set consists of mirror-reflected versions around the long axis of the reference pictures-abbreviated to $\mathrm{MrL}$. The pictures of the second set are $180^{\circ}$-rotated versions of the reference pictures (thus combination of mirror reflections around horizontal and vertical axis) - abbreviated to MrLS. In the third set, the comparison pictures differ from the reference pictures by a mirror reflection around the short
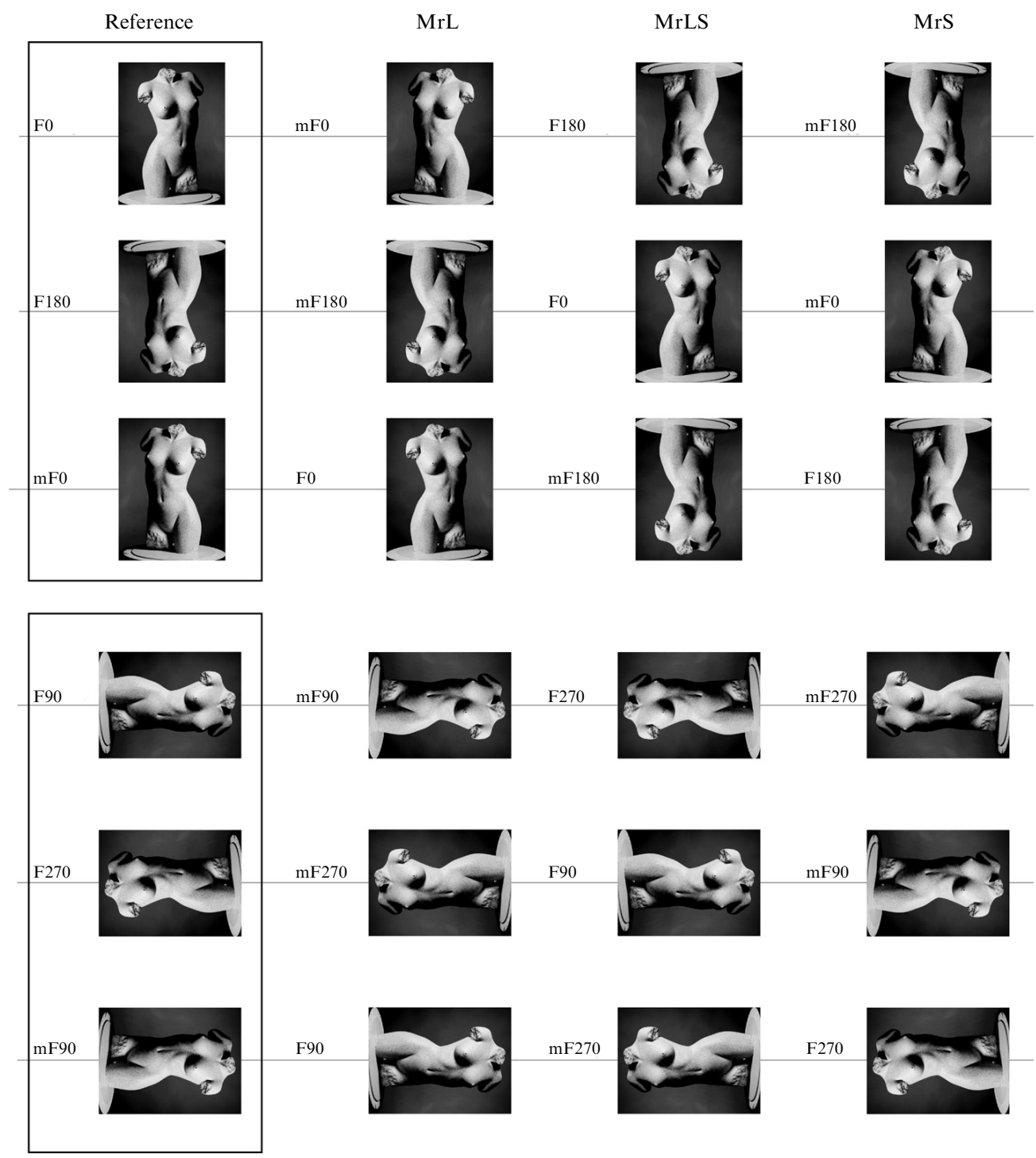

Figure 6. Complete grouping scheme of pictures based on one of the six original photographs (pose 060). The first column of pictures is the reference set. The second, third, and fourth columns are the three comparison sets. They consist of transformed versions of the reference pictures: $\mathrm{MrL}=$ mirror reflection around the long axis of the picture; $\mathrm{MrLS}=$ rotation of $180^{\circ}$; $\mathrm{MrS}=$ mirror reflection around the short axis of the picture. A distinction is made between the vertical and the horizontal picture orientation. F, fiducial; $\mathrm{m}$, mirror-reflected around long axis of the picture; 0 , not rotated; 90 , rotated by $90^{\circ} ; 180$, rotated by $180^{\circ} ; 270$, rotated by $270^{\circ}$. [The names of the pictures are originating from the production of the entire picture set as described in section 2 (see figure 2).] 
axis of the picture-abbreviated to MrS. In the following, we will use the abbreviations MrL, MrLS, and MrS to indicate the specific transformations. Furthermore, there is a distinction between the vertically (V) and the horizontally oriented pictures $(\mathrm{H})$, shown in the upper half and lower half of figure 6, respectively. The horizontally oriented picture set is exactly the same as the vertically oriented picture set, except for a rotation of $90^{\circ}$. The organisation of the pictures according to the transformation applied is thus consistent for both picture orientation sets. (Note that we have chosen to organise the set of comparisons in this way for the sake of clarity, although some comparisons occur more than once.)

\subsection{Results}

On the basis of the gauge-figure settings executed by the participants, pictorial reliefs were computed for all pictures. Comparisons were performed between the pictorial reliefs of the reference pictures and the pictorial reliefs of the accompanying comparison pictures for the vertically oriented picture set as well as for the horizontally oriented picture set, for each participant separately. Henceforth, comparison MrL (as well as MrLS and MrS) will refer to the comparison between the pictorial reliefs based on the reference picture and the respective comparison pictures MrL (as well as MrLS and MrS). Each time, the coordinate system was fixed to the reference picture: the $x$-dimension of the image plane of the reference picture was always the horizontal dimension (and $y$ was vertical). The coordinate system established for the reference picture was thus mirrored and rotated along with the accompanying comparison pictures.

The pictorial reliefs were compared by means of two analyses: (i) a straight regression (or correlation) analysis, relating the depth values of the comparison picture (comp) to the depth values of the reference picture (ref); and (ii) a multiple regression analysis with the depth values based on the reference picture and their corresponding $x, y$ image coordinates as predictors in order to predict the depth values based on the comparison picture. A straight regression analysis $z_{\text {comp }}=a+d z_{\text {ref }}$, with $z$ representing the depth values, accounts for possible depth-scaling effects between the pictorial relief of the reference picture and its transformed version. Depending on the slope of the regression line ( $d$-value), the pictorial relief based on the comparison picture is similar, flattened, or deepened compared to the pictorial relief from the reference picture. A multiple regression analysis, $z_{\text {comp }}=a+b x+c y+d z_{\text {ref }}$, with $z$ representing the depth values and $x$ and $y$ the image coordinates (measured in screen pixels and indicating the positions where the depth values were calculated), considers the depth $(z-)$ dimension, as well as the $x$ - and $y$-dimensions of the image plane. Geometrically, this multipleregression analysis accounts for a depth scaling and a shear between the pictorial reliefs of the reference picture and the comparison picture. Because of its geometrical properties (invariance of parallelism, collinearity, and ratios), this multiple regression is called an affine regression (Koenderink et al 2001). [For a non-technical discussion of invariant properties under different groups of transformations, see Van Gool et al (1994). For research showing the perceptual relevance of these affine invariant properties, see Kukkonen et al (1996) and Wagemans et al (1996, 2000).]

Scatterplots of the observed depth values of the reference picture versus the observed depth values of the comparison picture of EC, pose 060, for all comparisons as presented in figure 6 , are presented on the left side of figure 7 . The depth values were measured in screen pixels. The axes of the scatterplots range from -100 to +100 pixels. One can clearly notice that for some comparisons the linear relationships between the depth lists seem to be stronger than for other comparisons.

Examination of the $R^{2}$ values of the straight regression between the depth values based on the reference pictures and the depth values based on their comparison pictures, confirmed this observation (figure 8, black bars). Compared to the general range of $R^{2}$ values, 
particularly high $R^{2}$ values were found for comparison $\mathrm{MrL}$ in the set of vertically oriented pictures and for comparison $\mathrm{MrS}$ in the set of horizontally oriented pictures. As one can notice in the bar charts in figure 8, all other comparisons generally show rather small $R^{2}$ values.

F0
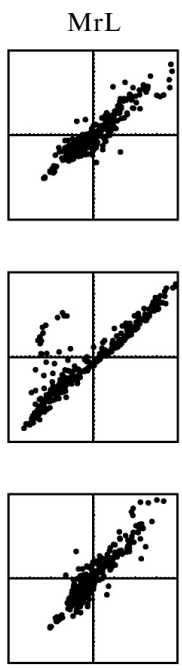

(a)
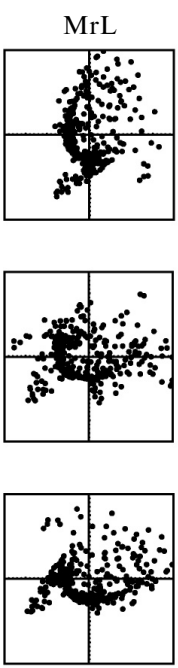

Vertically oriented picture set
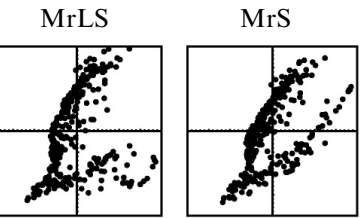

$\mathrm{MrL}$
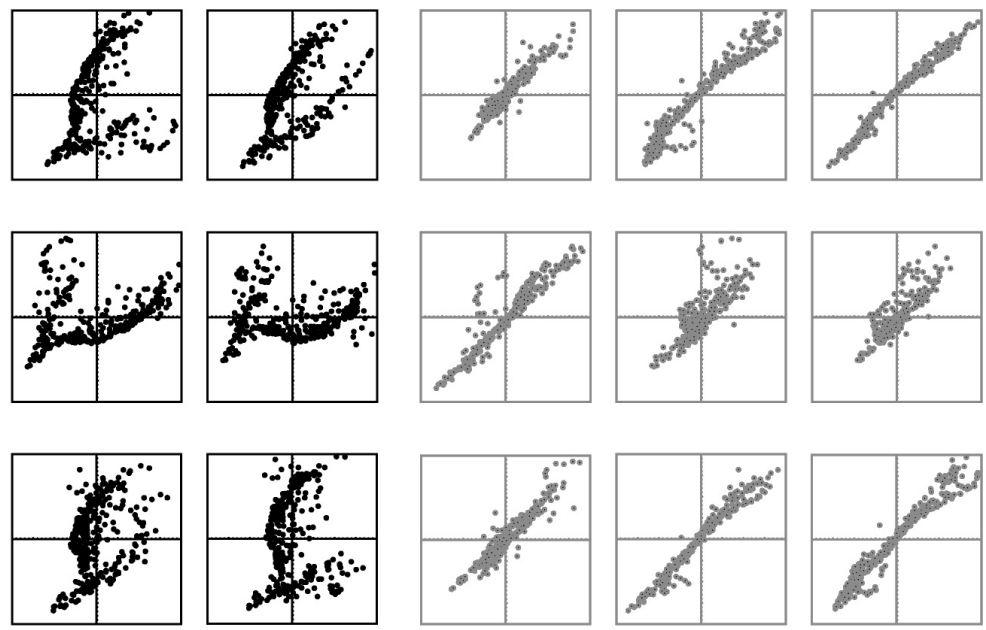

Horizontally oriented picture set

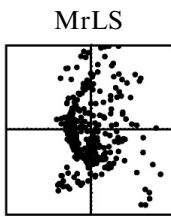

MrS
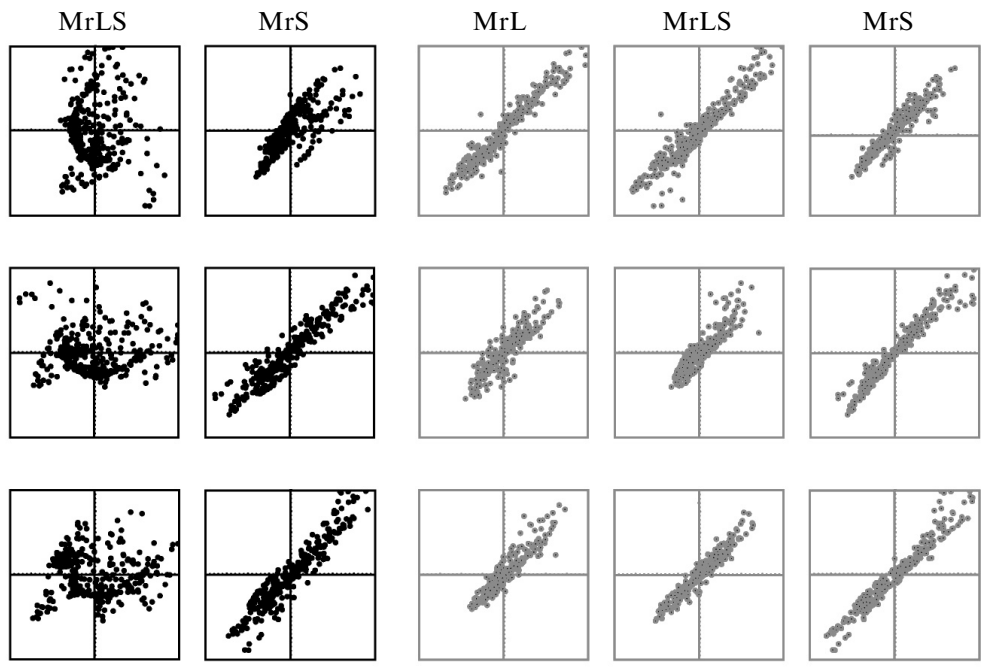

Figure 7. (a) On the left side (in black): scatterplots of the raw depth values obtained from the reference picture ( $x$-axis) versus the raw depth values obtained from the comparison picture ( $y$-axis). The depth values were measured in screen pixels. The axes range from -100 to +100 pixels. On the right side (in grey): scatterplots of the affinely corrected depth values from the reference picture $(x$-axis) versus the raw depth values obtained from the comparison picture ( $y$-axis). The depth values were measured in screen pixels. The axes range from -100 to +100 pixels. The depth values displayed were obtained on the basis of pose 060 from participant EC. The rows (columns) of scatterplots are consistent with the rows (columns) of pictures, as presented in figure 6. Each scatterplot in the first, second, and third column shows the relationship between the depth values of the reference picture and the comparison picture $\mathrm{MrL}$, MrLS, and $\mathrm{MrS}$, this for the vertically oriented picture set (a) as well as the horizontally oriented picture set (b). The reference picture of each row of scatterplots is indicated by F0, F180, mF0, F90, F270, and $\mathrm{mF} 90$. 
Vertically oriented picture set

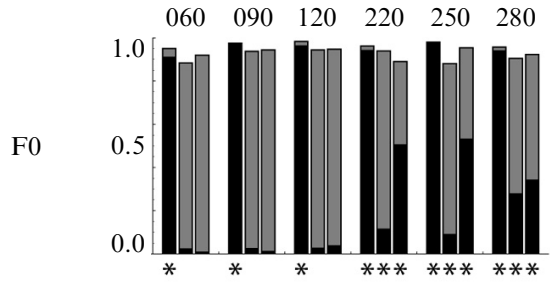

$\begin{array}{llllll}060 & 090 & 120 & 220 & 250 & 280\end{array}$
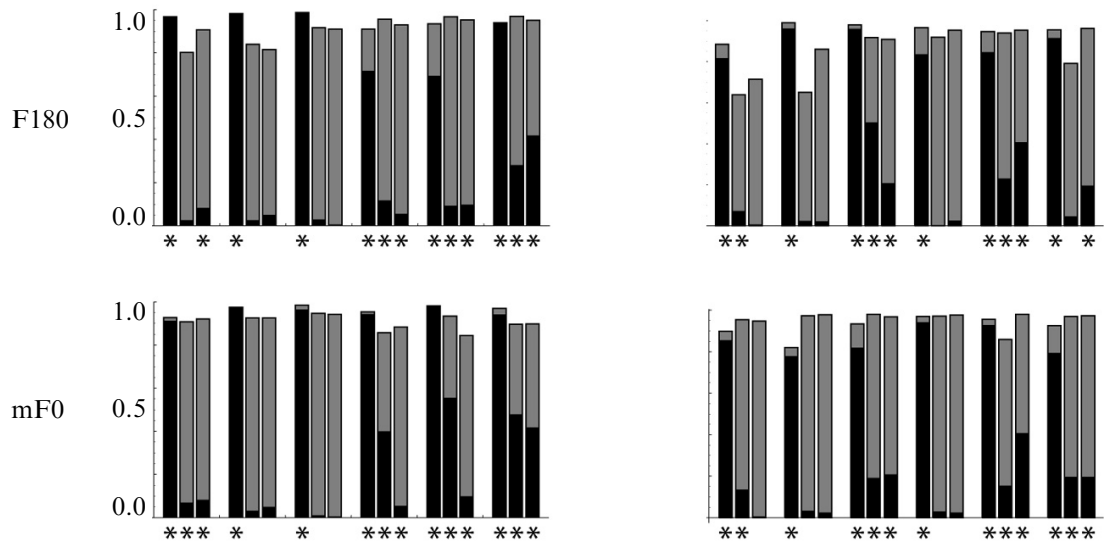

Horizontally oriented picture set

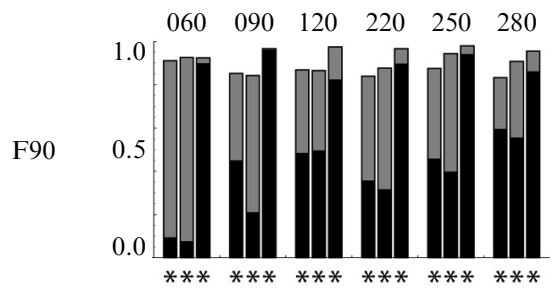

$\begin{array}{llllll}060 & 090 & 120 & 220 & 250 & 280\end{array}$
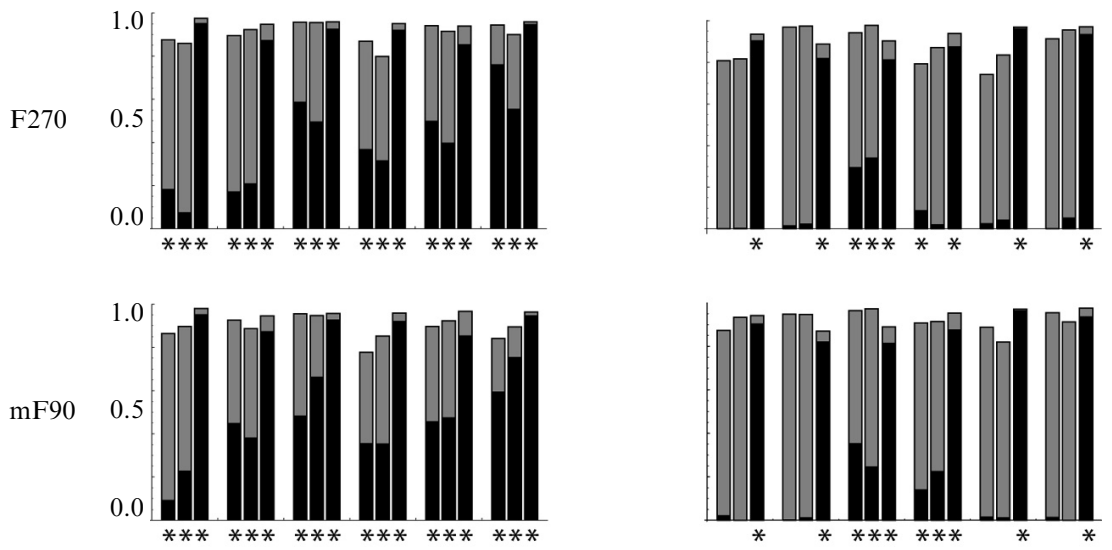

Figure 8. Bar charts showing the $R^{2}$ values of the straight (black bar) and affine regression (grey bar) obtained from AD (on the left) and EC (on the right). Each group of three refers to one pose $(060,090,120,220,250$, and 280). Every bar of each group of three refers to one comparison, ie comparison $\mathrm{MrL}$, comparison MrLS, and comparison MrS. The bar charts on top (at the bottom) of the figure are related to the comparisons with the vertically (horizontally) oriented picture set. Each time, the reference pictures of the comparisons are specified by F0, F180, mF0, F90, F270, and mF90. Significant $R^{2}$ values of the straight regression are indicated by * $(p<0.001)$; the $R^{2}$ values of the affine regression were all significant $(p<0.0001)$. 
Multiple regression analyses relating the depth values from the comparison picture with the $x, y$ image coordinates and the depth values based on the reference picture, showed generally high $R^{2}$ values (figure 8 , grey bars). In all cases, a statistically significant gain $(p<0.01)$ in proportion of explained variance was obtained with the affine regression model compared to the straight regression. Except for comparison $\mathrm{MrL}$ in the vertical picture set and comparison $\mathrm{MrS}$ for the horizontal picture set, the gain between straight and affine regression was substantial (compare black and grey bars in figure 8). This is also clearly noticeable when comparing the affinely corrected scatterplots (on the right side of figure 7) with the raw scatterplots (on the left side of figure 7). The scattterplots representing the affinely transformed depth values of the reference pictures versus the observed depth values of the comparison pictures show strong linear trends for all comparisons. Moreover, when the depth values of the reference picture are transformed affinely towards the observed depth values of the comparison picture, the chaotic pattern observed in the raw scatterplots of comparisons MrLS and MrS for the vertical orientation and comparisons MrL and MrLS for the horizontal orientation, almost completely disappeared.

In the case of comparison MrLS and MrS for the vertical orientation and comparison MrL and MrLS for the horizontal orientation, straight regression (and thus depth scaling) did not suffice to explain the variance in the data. Since including the image plane coordinates as predictors in the regression model resulted in high $R^{2}$ values, we may assume that shears largely explain the differences between the pictorial reliefs. We calculated the shears occurring between the pictorial relief of the comparison picture and the pictorial relief of the reference picture on the basis of the weights of the image coordinates in the affine regression (see caption of figure 9 for more details). In figure 9, polar representations of the shears are shown for $\mathrm{AD}$ and $\mathrm{EC}$ for all comparisons and for all poses. It is interesting to note that the magnitudes and the directions of the shears observed were highly similar over the various poses used. Considering the magnitudes of the shears, it is obvious that comparison MrL for the vertically oriented pictures and comparison $\mathrm{MrS}$ for the horizontally oriented pictures demonstrated negligible shears. In these cases, the direction of the shear is of no importance. However, it is interesting to consider the direction of the shears for comparison MrLS and MrS for the vertical orientation and for comparison MrL and MrLS for the horizontal orientation (figure 9). It can be noticed that the direction of the shear for comparison MrL and MrLS for the horizontally oriented pictures is in the negative vertical direction, meaning that the upper part of the pictorial relief based on the reference picture needed a slant correction to the front in order to fit the pictorial relief of the comparison picture (and, likewise, to the back for the lower part). For the vertically oriented pictures, the direction of the shear for the MrLS and the $\mathrm{MrS}$ comparisons is in the negative vertical direction as well. [Note that the dimensions of each shear graph are consistently related to the dimensions of the image plane of the reference picture. The horizontal dimension of each shear graph is corresponding with the $x$-dimension of the image plane of the reference picture (and the vertical axis with the $y$-dimension), whatever the particular orientation of the reference picture may be.]

\subsection{Discussion}

The main question we addressed until now was: what are the effects of the various mirror reflections applied to the picture on the shape percept of the depicted 3-D object? The transformations examined were mirror reflections around the long $(\mathrm{MrL})$ and short axis $(\mathrm{MrS})$ of the picture frame and a $180^{\circ}$ rotation of the picture (a combination of both mirror reflections-MrLS). And related to the main question: would the transformations applied to the picture have the same effect independently of the picture's orientation? 

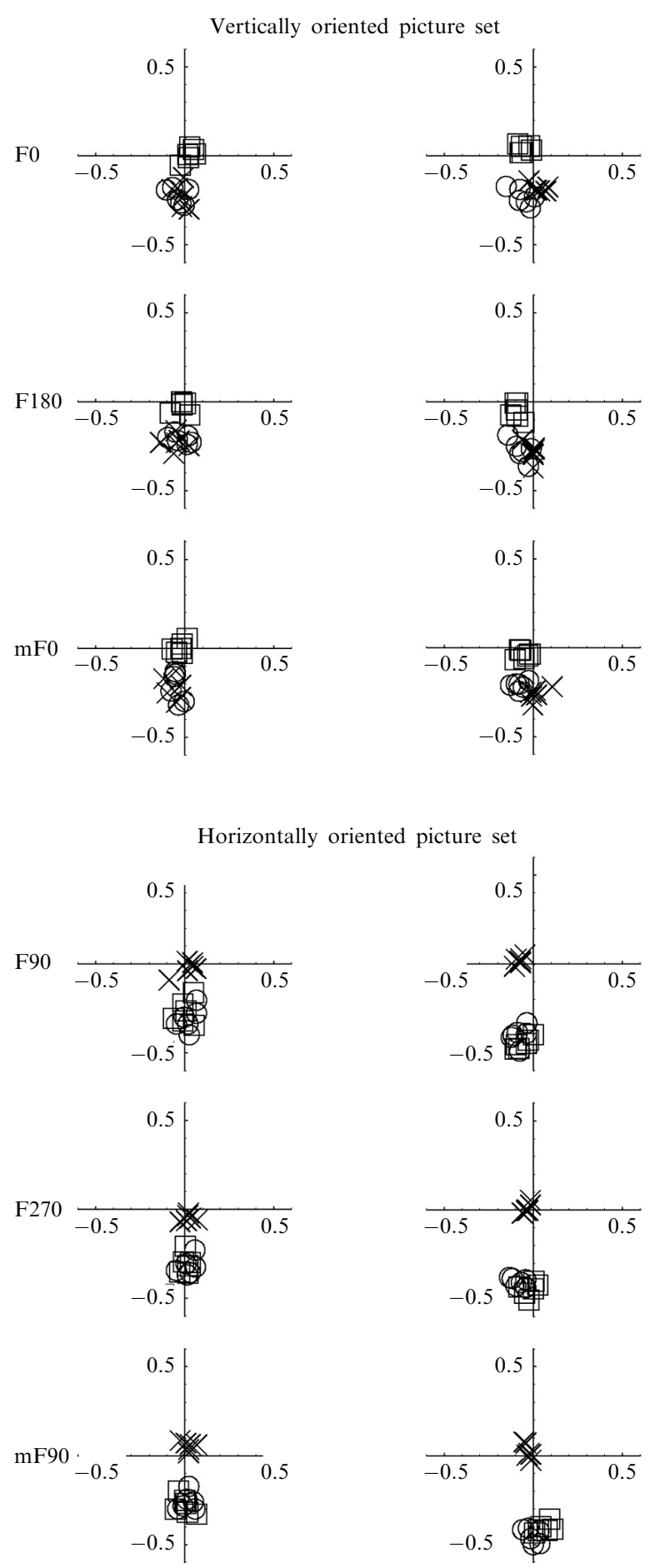

Figure 9. Polar representation of the shears between the pictorial reliefs of the reference pictures and the comparison pictures, obtained from AD (on the left) and EC (on the right). We calculated the shears on the basis of the weights $b$ and $c$ of the image coordinates $x$ and $y$ in the regression model. The arctangents of the ratio of the weights $c$ and $b$ defines the direction of the shear; a measure for its magnitude was obtained by computing $\sin \left\{\arctan \left[\left(b^{2}+c^{2}\right)^{1 / 2}\right]\right\}$, thereby limiting the range from 0 to 1 . Both axes of the shear graph range from -0.6 to +0.6 , thereby only presenting a portion of the total range $(-1$ to +1$)$. The orientation of the shear graph is consistently related to the orientation of the pictorial relief based on the reference picture, indicated by $\mathrm{F} 0, \mathrm{~F} 180, \mathrm{mF} 0$, F90, F270, and mF90. The horizontal (vertical) dimension of the shear graph is corresponding with the horizontal dimension (vertical) of the image plane of the reference picture. All six poses are displayed in each shear graph. MrL, MrLS, and MrS refer to the different comparisons with the reference picture. 
We have found that the pictorial reliefs of comparison $\mathrm{MrL}$ for the vertically oriented pictures as well as the pictorial reliefs of comparison $\mathrm{MrS}$ for the horizontally oriented pictures were highly similar. All other comparisons (comparisons MrLS and $\mathrm{MrS}$ in $\mathrm{V}$; comparisons $\mathrm{MrL}$ and $\mathrm{MrLS}$ in $\mathrm{H}$ ) clearly showed dissimilarities between the pictorial reliefs. When affinely correcting the depth values of the reference pictures towards the depth values of the comparison pictures, the dissimilarities almost completely disappeared, indicating that shears could largely account for the differences observed between the pictorial reliefs.

Although, considered from the dimensions of the picture, the transformations were the same for both picture orientation sets, the effects of transformation MrL and transformation $\mathrm{MrS}$ were found to be different depending on picture orientation. Taking into account an external reference frame (eg left, right, top, bottom), transformation $\mathrm{MrL}$ in $\mathrm{V}$ and transformation $\mathrm{MrS}$ in $\mathrm{H}$ can be summarised as mirror reflections around the vertical axis. Transformation $\mathrm{MrS}$ in $\mathrm{V}$ and transformation $\mathrm{MrL}$ in $\mathrm{H}$ can be described as mirror reflections around the horizontal axis. Mirrorreflecting pictures around the vertical axis thus seems to have had little influence on the shape perception of the depicted object. Up-down mirror-reflecting of pictures and mirror reflection around both axes showed much larger effects.

3.3.1 Mirror reflection around the vertical axis. In the present research, the pictorial reliefs of the left-right mirror-reflected versions were found to be similar to the pictorial reliefs of the reference pictures, for the vertically oriented pictures as well as for the horizontally oriented pictures. In Cornelis et al (2003), the vertical orientation of the depicted torso was suggested as a possible reason underlying the similarity between the pictorial reliefs of the original (straight up, vertically oriented) photograph and the left-right mirror-reflected version. Since the torso has a strong intrinsic vertical axis, it would have been possible that a left - right mirror reflection of the original photograph would have had little effect, in contrast to a transformation that would interchange top and bottom (such as an up-down mirror reflection). In the present study, however, for the horizontally oriented pictures (with horizontally oriented torsos and thus strong horizontal intrinsic axes), not the pictorial reliefs of the up-down mirror-reflected versions but again those of the left-right mirror-reflected versions showed larger similarity with the pictorial reliefs of their respective reference pictures. This finding suggests that the type of transformation, considered from an absolute reference frame, is largely determining the consequences on shape perception.

Shape perception of a depicted object thus seems to be largely symmetrical when presented on pictures differing from each other by mirror reflection around the vertical axis. This is an interesting observation considering the ecological value vertical symmetry is thought to have in the natural world (Moeller and Thornhill 1998; Perrett et al 1999; Rhodes et al 1998) as well as the artificial world. Additionally, empirical research on symmetry perception has generally provided evidence in favour of the special status of vertical symmetry compared to horizontal or diagonal symmetry (see Wagemans 1995 for a review). Goldmeier (1936/1972), for instance, asked participants to choose which comparison figure (a vertically or a horizontally symmetrical one) was more similar to the double-symmetrical reference figure and found that the vertically symmetrical figure was chosen more frequently. Other studies focused on the detectability of symmetry in patterns. Vertical symmetry was found to be detected faster than horizontal symmetry (Corballis and Roldan 1975; Evans et al 2000; Palmer and Hemenway 1978; Wagemans et al 1992; Wenderoth 1996; Zimmer 1984), as well as more accurately (Barlow and Reeves 1979; Evans et al 2000; Wagemans et al 1992; Wenderoth 1994, 1996). Furthermore, this perceptual advantage seems to occur already very early in development: 4-month-old infants processed vertically symmetrical patterns more efficiently, ie more 
quickly and completely, than horizontally symmetrical ones (Bornstein et al 1981; Bornstein and Krinsky 1985).

Research on symmetry perception as described above has generally used stimulus material consisting of 2-D pictures of 2-D patterns, 2-D configurations, or 2-D figures that were somehow symmetrical to a smaller or larger degree. In the present study, symmetry was investigated in a completely different way, not only by means of different stimulus material-ie 2-D pictures of 3-D objects - but also by applying a different experimental method. The pictures and their transformed versions were not shown side by side on a computer screen but at different moments in time. Furthermore, the six pictures and their various transformations were presented in random order. Additionally, the complete stimulus set was enormous: in total it contained 48 presentations of pictures and the task was performed on each picture three times. It would thus have been extremely hard to find out which picture was the transformed version of which picture while running the experiment. Moreover, participants were not asked to compare pairs of stimuli and thus to judge similarity in their shape percepts: they were only asked to set a local gauge figure onto the depicted object. From their settings, a pictorial relief was constructed for each picture. This way, we were able to quantitatively compare the pictorial reliefs (percepts) on the basis of the reference pictures and the transformed versions. Perceptual similarity between figures (ie depicted torsos) was thus investigated from quite a different starting point than in other studies on this issue. All the same, we still have found evidence supporting the special status of vertical symmetry over horizontal symmetry or rotation around $180^{\circ}$ : the pictorial reliefs of the left-right mirror-reflected pictures showed strong similarity with the pictorial reliefs of the reference pictures.

3.3.2 Mirror reflection around the horizontal axis and $180^{\circ}$ rotation. The pictorial reliefs of the pictures that were mirror-reflected around the horizontal axis or $180^{\circ}$ rotated showed large and complex differences from the pictorial reliefs of their reference pictures. Pictures that were mirrored upside down showed pictorial reliefs that differed by shears from the pictorial reliefs based on their respective reference pictures (see also Cornelis et al 2003). Moreover, the direction and the magnitude of these shears were very similar to the ones observed for the $180^{\circ}$ rotations. In both cases, the shear between the pictorial reliefs of the reference picture and the manipulated picture were in the vertical direction. More specifically, the upper part of the pictorial relief based on the reference picture had to be slanted frontwards to fit the pictorial relief of the comparison picture. We included the $180^{\circ}$ rotation in the set of transformations, since it can be regarded as a combination of mirror reflections around the vertical axis and the horizontal axis. The similarity between the effects of the $180^{\circ}$ rotation and the up-down mirror reflections suggested that there was no interaction effect between the two mirror-reflection components in the $180^{\circ}$ rotation. This is not that surprising anymore since we already observed that mirror reflections around the vertical axis gave rise only to minimal effects for all the comparisons and a $180^{\circ}$ rotation and an up-down mirror reflection are differing by a left-right mirror reflection.

The similar effect of $180^{\circ}$ rotation and mirror reflection around the horizontal axis was not found to be supported in the symmetry literature. Detection of symmetry in a rotational symmetrical pattern/figure - a pattern or figure in which one half is $180^{\circ}$ rotated - was for instance reported to be less easily perceivable than symmetry around the horizontal or vertical axis (Julesz 1971; Palmer and Hemenway 1978; Royer 1981). Furthermore, using flat random figures as illustration, Mach $(1886 / 1959)$ argued that there is perceptual similarity between left-right mirror-reflected versions or $180^{\circ}$-rotated versions with their original counterparts, but not between up-down mirrored images. Our findings partly contradict his observations: $180^{\circ}$ rotation seems 
to have perceptual consequences on the 3-D shape percept of the depicted objectsimilar to the perceptual consequences of the mirror reflection around the horizontal axis; the perceptual consequences of mirror-reflecting a picture around the vertical axis seem to be much less significant.

Furthermore, the effect of presenting pictures upside down on the shape percept of the depicted object was established in the vertically oriented picture set as well as in the horizontally oriented picture set. In our previous study (Cornelis et al 2003) the pictorial reliefs of the mirror-reflected versions were compared with the pictorial reliefs of the original (and thus straight up) photographs. Mirror-reflecting a picture around the horizontal axis resulted in different pictorial reliefs compared to the pictorial reliefs obtained from the original photograph. This effect could have been attributable to two main variables. First, there was, of course, the transformation itself, ie mirror reflection around the horizontal axis. Second, the photograph depicted a female torso, which was lit from left above: it was thus a natural scene including a meaningful object that had a strong intrinsic axis. Presenting such a photograph, and thus the natural scene together with the direction of the light source, upside down could be considered as rather unnatural. If the latter had been of importance, different results would have been found for the up-down mirror reflection for the vertical and the horizontal picture set since in the horizontal picture set, the upside-down versions were not more or less natural than their reference counterparts. However, presenting a picture upside down resulted in large pictorial-relief differences for the vertically oriented picture set as well as for the horizontally oriented picture set. This leads us to conclude that the transformation itself, ie showing a picture upside down by mirror reflection around the horizontal axis or by a $180^{\circ}$ rotation, is the main contributing factor to the shape perception differences found.

As has already been mentioned, the differences between the pictorial reliefs of the reference pictures and their upside-down-presented versions could be largely ascribed to shears. The shears observed can be considered as global attitude changes. A possible interpretation for the attitude changes observed between pictorial reliefs is that a change in mental viewpoint takes place when presented pictures upside down (for similar mental viewpoint interpretations, see Cornelis et al 2003; Koenderink et al 2001). It is obvious that the depicted objects cannot change orientation-the picture itself stays the same. The attitude change between pictorial reliefs indicates that different points are taken to be frontoparallel. It is as if the observer enters the pictorial space and looks at the object from a viewpoint differing from the actual, physical viewpoint, thereby 'seeing' different surface patches being frontoparallel. The picture ambiguities allow the observer to access various interpretations, within the constraints imposed by the cues given in the picture. Following Ernst Gombrich (1977), Koenderink and van Doorn (2003) called this 'the beholder's share'. Interestingly, observers looking at pictures in an upside-down view resolve the ambiguities in the picture differently than when looking at the reference pictures. Hence, the beholder's share differs between these conditions.

\section{The effect of rotation of pictures on the shape percept of the depicted object}

The second research question addressed the effects of various degrees of rotation of pictures on the shape percept of the depicted object. More specifically, we investigated the effects of rotations of pictures by (i) $90^{\circ}$, (ii) $180^{\circ}$, and (iii) $270^{\circ}$.

Rotating a rectangular framed picture by $90^{\circ}$ or $270^{\circ}$ always results in a different picture-frame orientation compared to the picture-frame orientation of the reference picture. When the reference picture is oriented vertically (ie the long axis of the frame is vertical), the $90^{\circ}$ - or $270^{\circ}$-rotated versions are oriented horizontally and vice versa. 
Moreover, it is obvious that not only the frame format is different when having rotated the reference pictures by $90^{\circ}$ or $270^{\circ}$ : also the intrinsic axes of the object, the torso, depicted in the picture drastically change orientation. We were thus particularly interested in observing the effect of rotations of pictures around $90^{\circ}$ and $270^{\circ}$ on the shape percept. Would the effect of a $90^{\circ}$ or $270^{\circ}$ rotation be larger compared to the effect of a $180^{\circ}$ rotation - as Mach $(1886 / 1959)$ would have assumed? Or would they be smaller since the angle of rotation relative to the vertical was smaller, ie a $90^{\circ}$ rotational difference in contrast to a $180^{\circ}$ rotational difference?

Furthermore, we were interested in the nature of the differences between the pictorial reliefs of the reference pictures and the pictorial reliefs of the $90^{\circ}$ - or $270^{\circ}$-rotated versions: could they be described by depth scaling and shears as well? And, if so, how would the direction of the shear behave?

In this respect, it is noteworthy referring to the mental rotation literature, reporting larger reaction times to identify figures rotated by $180^{\circ}$ than figures rotated by $90^{\circ}$ (Shepard and Cooper 1982). It is, however, difficult to derive clear predictions from the mental rotation studies for the present study, since, as has been mentioned above, the approaches of both are of a completely different nature. In the present study, participants were not asked to identify or to compare the pictures shown at different moments in time. They were only instructed to perform a simple, visual task: adjusting a gauge figure so that it seemed to fit onto the depicted object.

\subsection{Organisation of stimuli}

In analogy with the first part of this research, in which mirror reflections were studied, we investigated the effect of the transformation itself-here rotation-on the shape percept of the depicted object. Hereto, we included not only the pictorial relief comparisons between the original photographs and the manipulated pictures, but we also performed every possible comparison between two pictorial reliefs based on pictures that differed from each other by a $90^{\circ}, 180^{\circ}$, or $270^{\circ}$ rotation. Hence, we organised the complete picture set such that each picture of the picture set was selected once as a reference picture. The accompanying comparison pictures, grouped according to the various degrees of rotation, were (i) the $90^{\circ}$-rotated versions (Rot90); (ii) the $180^{\circ}$ rotated versions (Rot180); and (iii) the $270^{\circ}$-rotated versions (Rot270) of the reference picture. A complete grouping scheme on the basis of one of the six original photographs (pose 060) is presented in figure 10. As before, several reference-picture-comparisonpicture combinations occur more than once for reasons of clarity.

\subsection{Results}

The influence of rotation of the picture on the 3-D interpretation of the depicted object was investigated by comparing the pictorial reliefs of the reference pictures with the pictorial reliefs of their rotated counterparts.

The straight regression analyses performed on the depth values of the rotated pictures and the depth values of the reference pictures revealed $R^{2}$ values ranging from 0.008 to 0.871 for $\mathrm{AD}$, from 0.000 to 0.719 for $\mathrm{EC}$, from 0.163 to 0.936 for HR, and from 0.000 to 0.759 for $\mathrm{KJ}$. In figure 11 , the $R^{2}$ values for the straight regression for $\mathrm{AD}$ and EC are presented by the black bars in the bar charts.

The $R^{2}$ values obtained by performing the multiple regression analyses (with the image plane coordinates and the depth values of the reference picture as predictors) were high. The smallest $R^{2}$ values were $0.773,0.638,0.698$, and 0.798 for AD, EC, HR, and KJ, respectively, while the highest $R^{2}$ values were $0.982,0.985,0.967$, and 0.967 for $\mathrm{AD}, \mathrm{EC}, \mathrm{HR}$, and $\mathrm{KJ}$, respectively. Nearly all variance between the pictorial reliefs of the reference pictures and the rotated pictures thus seemed to be explained by the affine regression. Moreover, in all cases statistically significant gains $(p<0.01)$ were obtained when comparing the $R^{2}$ values of the straight regression analysis with the $R^{2}$ 

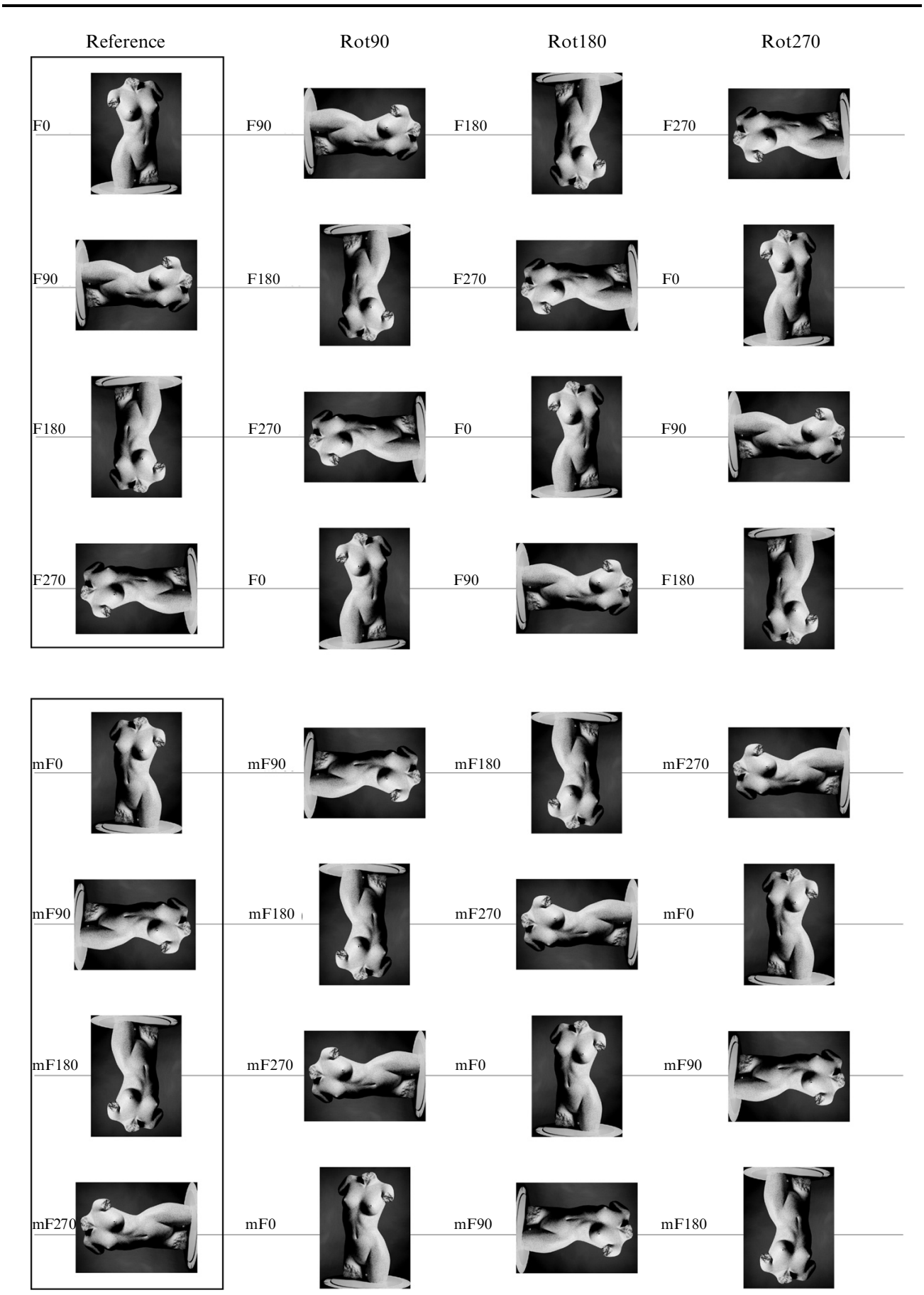

Figure 10. Complete grouping scheme of pictures based on one of the six original photographs (pose 060). The first column of pictures is the reference set. The second, third, and fourth columns represent the three comparison sets. They consist of transformed versions of the reference pictures: the first transformation is the rotation by $90^{\circ}$ (Rot90), the second the rotation by $180^{\circ}$ (Rot 180 ), and the third the rotation by $270^{\circ}$ (Rot270). The upper group of pictures is based on the original picture and its rotated versions; the lower group of pictures is based on the left - right mirror-reflected version and its rotated versions. $\mathrm{F}$, fiducial; $\mathrm{m}$, mirror-reflected around long axis of the picture; 0 , not rotated; 90 , rotated by $90^{\circ} ; 180$, rotated by $180^{\circ} ; 270$, rotated by $270^{\circ}$. [The names of the pictures are originating from the production of the entire picture set as described in section 2 (see figure 3).] 

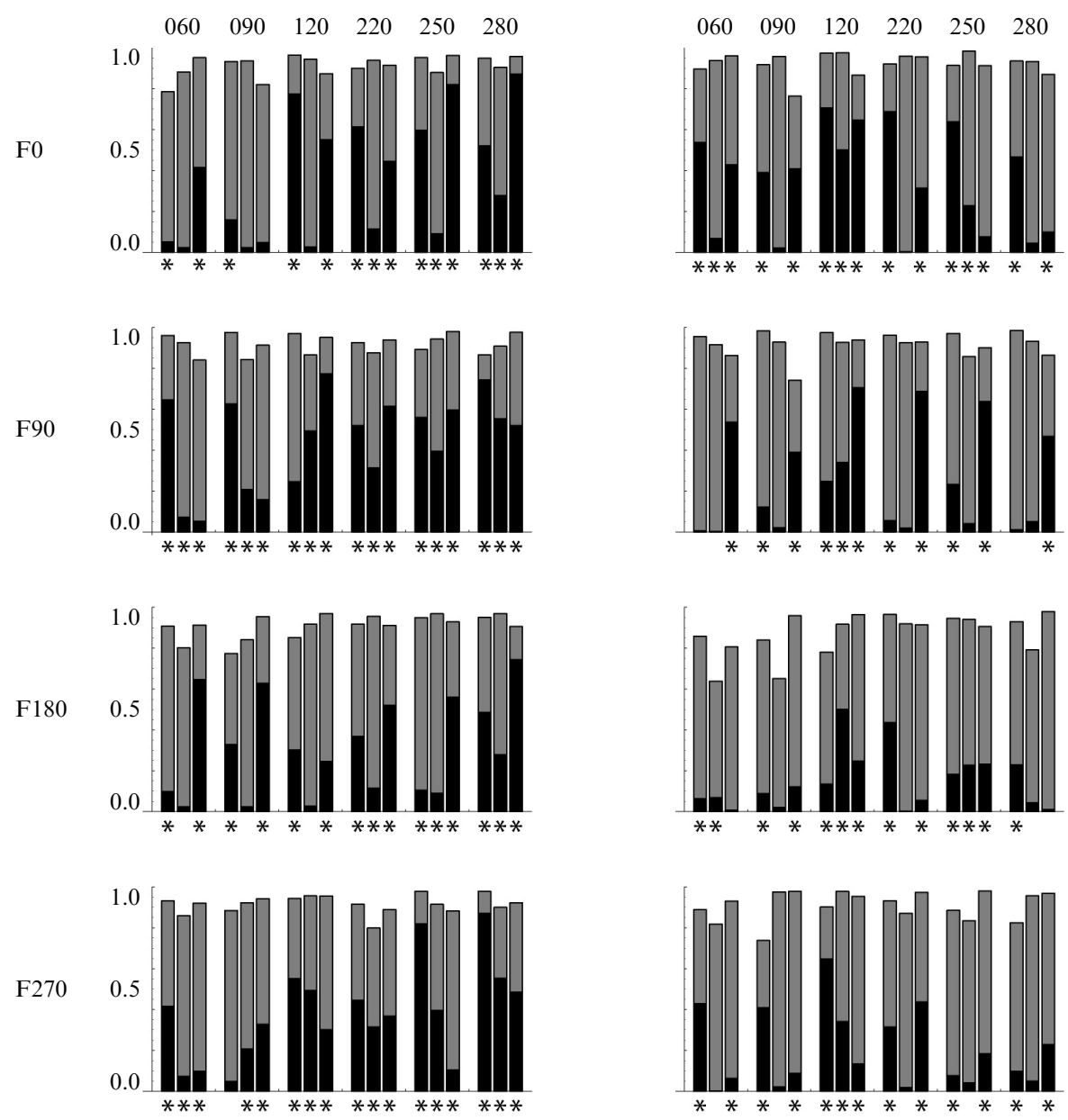

Figure 11. Bar charts showing the $R^{2}$ values of the straight (black bar) and affine regression (grey bar) obtained from AD (first column) and EC (second column). Each group of three refers to one pose $(060,090,120,220,250$, and 280). Every bar of each group of three refers to one comparison, ie comparison Rot90, comparison Rot180, and comparison Rot270. The bar charts are related to the various comparisons performed, as is shown in figure 10. Each time, the reference pictures of the comparisons are specified by F0, F90, F180, F270, mF0, mF90, $\mathrm{mF} 180$, and $\mathrm{mF} 270$. Significant $R^{2}$ values of the straight regression are indicated by * $(p<0.0001)$; the $R^{2}$ values of the affine regression were all significant $(p<0.0001)$.

values of the multiple regression analysis. In general, the gain between the two models was substantial. In figure 12, some raw scatterplots as well as the corresponding affinely corrected scatterplots are demonstrated for pose 280, reference picture mF90, participant HR. Clearly, the raw scatterplots depicting the observed depth values of a reference picture versus the observed depth values of its rotated version demonstrate more or less scattered data. However, in the affinely corrected scatterplots, the linear relationship between the affinely corrected depth values based on the reference picture and the observed depth values of the rotated picture shows to be strong.

As mentioned above, the affine regression did not only consider the depth dimension ( $z$-dimension) but also the image plane dimensions ( $x$-dimension and $y$-dimension), thereby accounting for not only possible depth scaling but also global attitude changes between pictorial reliefs. The magnitudes of the shears, averaged over poses, tended to be higher for the Rot180 comparisons than for the Rot90 and Rot270 comparisons. 

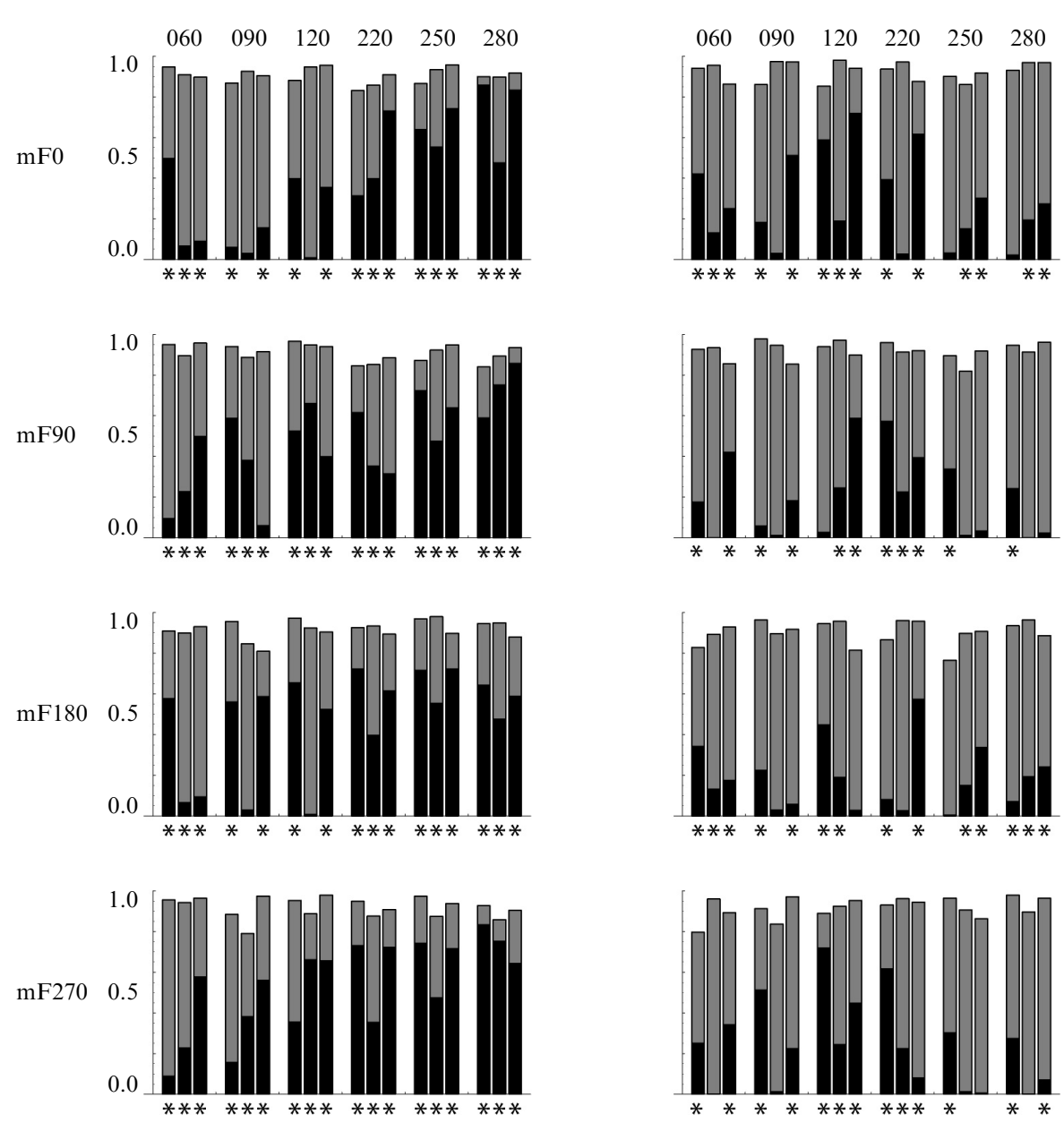

Figure 11 (continued)

In figure 13, the shears between the pictorial reliefs of the reference pictures and the comparison pictures obtained for AD and EC are graphically shown in polar coordinate representations. The direction of the shear was systematically related to the degree of rotation. The rotation of $180^{\circ}$ clearly showed a shear in the negative vertical direction: as mentioned before, this means that the top part of the pictorial relief of the reference picture had to be slanted to the front in order to obtain the pictorial relief of the Rot180 picture (and to the back for the bottom part). Compared to the Rot180 comparison, the Rot90 and Rot270 comparisons generally showed shears occurring somewhat less in the vertical direction but more in the horizontal direction. As can be noticed in figure 13, the Rot90 comparisons are mainly situated on the left side of the vertical axis of the graph. This indicates that the right side of the pictorial relief based on the reference picture came forward in order to become similar to the pictorial relief of the Rot90 picture (and backward for the left side), in addition to the shear in the vertical direction. The Rot270 comparisons, on the other hand, occurred at the bottom-right part of the graph, signifying that the left side and the top part of the pictorial relief based on the reference picture had to be slanted towards the observer to approximate the pictorial relief of the Rot270 pictures (and vice versa for the bottomright parts). The general directions of the shears were observed consistently over the various poses as well as the specific reference-comparison picture combinations and participants. 


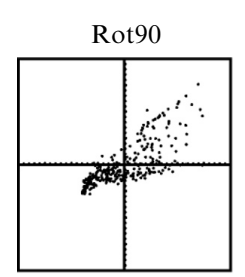

(a)

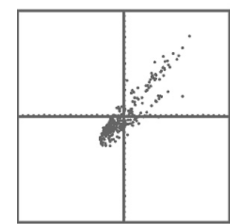

(b)
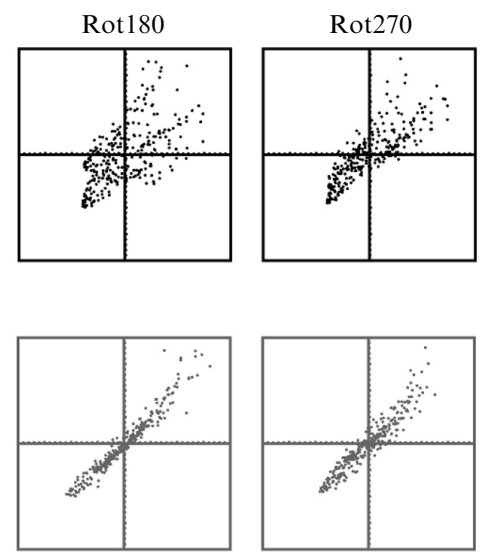

Figure 12. (a) Scatterplots of the raw depth values obtained from the reference picture (x-axis) versus the raw depth values obtained from the comparison pictures Rot90, Rot180, and Rot270 ( $y$-axis). The depth values were measured in screen pixels. The axes range from -100 to +100 pixels. (b) Scatterplots of the affinely corrected depth values from the reference picture ( $x$-axis) versus the raw depth values obtained from the comparison picture $\operatorname{Rot} 90, \operatorname{Rot} 180$, and $\operatorname{Rot} 270$ ( $y$-axis). The depth values were measured in screen pixels. The axes range from -100 to +100 pixels. The depth values displayed were obtained on the basis of the $280^{\circ}$ pose from participant HR. The reference picture of the comparison was mF90.

\subsection{Discussion}

In order to investigate the effect of rotation, we compared the pictorial reliefs derived from pictures, based on the same photograph, but differing by a $90^{\circ}$ (Rot90), $180^{\circ}$ (Rot180), or $270^{\circ}$ (Rot270) rotation. Generally, all comparisons showed that the pictorial reliefs of the rotated pictures were different from the pictorial reliefs of the reference pictures. Moreover, here again, straight regression-accounting for possible depth scaling only-did not suffice to describe the observed differences. Affine regressions, however, could explain nearly all variance, indicating that there were global attitude differences (shears) between the pictorial reliefs of the reference pictures and their rotated counterparts. For all comparisons, the direction of the shear between the pictorial reliefs of the reference and the rotated pictures could be summarised as follows: each time, the top side of the pictorial relief based on the comparison picture (with the pictorial relief having the same orientation as the comparison picture on which it is based) was slanted frontwards compared to the pictorial relief based on the reference picture. In addition, referred to the pictorial reliefs of the reference pictures, in all cases shearing took place in the vertical direction: the top part of the pictorial reliefs of the reference pictures had to be slanted to the front to become equivalent to the pictorial reliefs of the $90^{\circ}$ - and $270^{\circ}$-rotated versions.

Furthermore, the behaviour of the shear was found to be consistent over the various combinations of reference pictures with their rotated versions. This general trend was thus not limited to the comparison between the pictorial reliefs based on the original (or its mirror-reflected picture) and the pictorial reliefs based on the rotated versions. Moreover, similar shears were found for the comparisons including the more 'peculiar' views of the object regarding not only the more visual characteristics (eg the lighting direction with the accompanying shading pattern on the depicted object) but also the more cognitive aspects (eg familiarity of a view of the specific depicted object). Although it is possible that the variations in the data (ie magnitude/direction of the shear) were related to one of those, both, or other aspects (such as the different poses in which the object was photographed), the general trend thus seemed to depend largely on the transformation itself. 

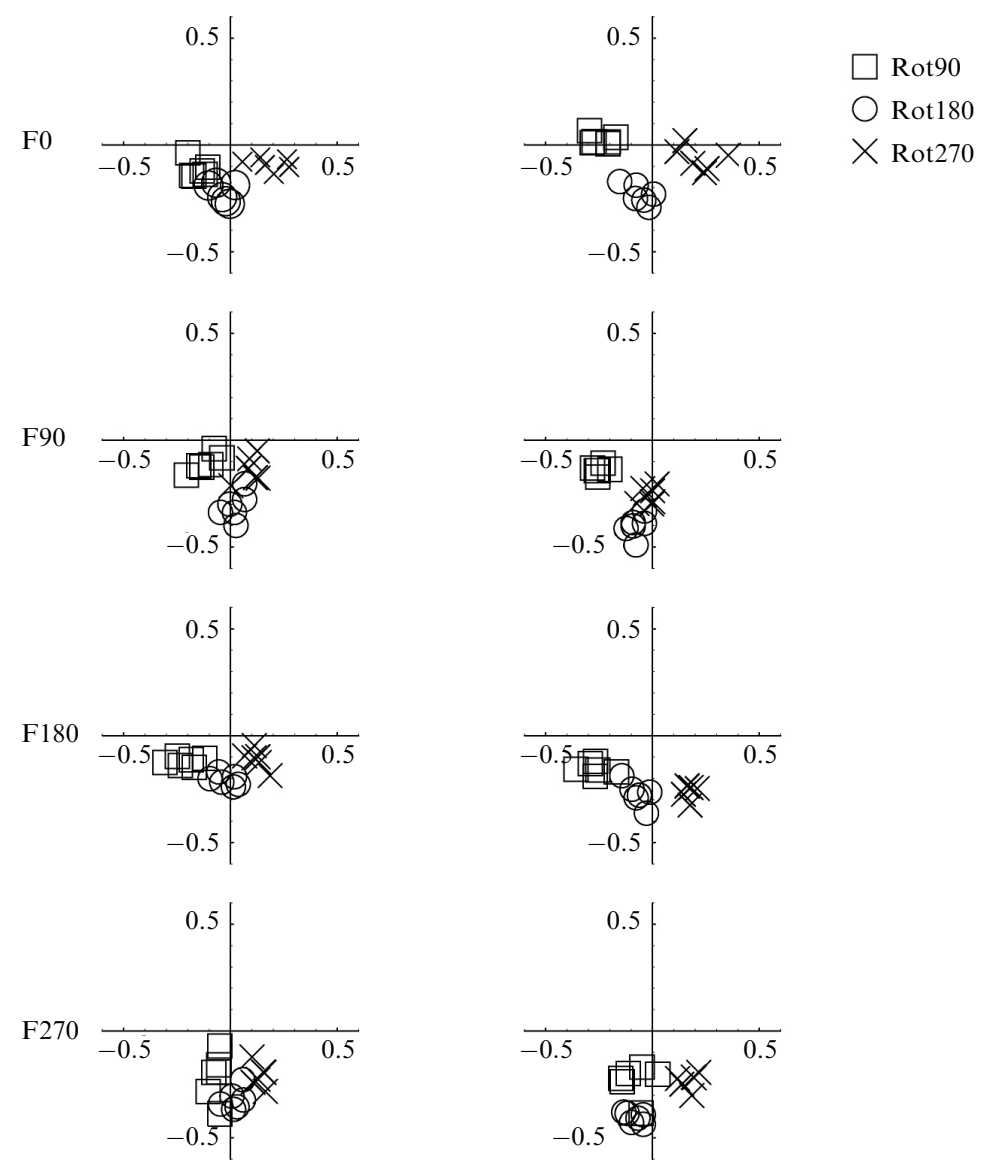

Figure 13. Polar representation of the shears between the pictorial reliefs of the reference pictures and the comparison pictures obtained from AD (on the left) and EC (on the right). We calculated the shears on the basis of the weights $b$ and $c$ of the image coordinates $x$ and $y$ in the regression model. The arctangents of the ratio of the weights $c$ and $b$ defines the direction of the shear; a measure for its magnitude was obtained by computing $\sin \left\{\arctan \left[\left(b^{2}+c^{2}\right)^{1 / 2}\right]\right\}$, thereby limiting the range from 0 to 1 . Both axes range from -0.6 to +0.6 , thereby only presenting a portion of the total range $(-1$ to +1$)$. The orientation of the shear graph corresponds with the orientation of the pictorial relief based on the reference picture, indicated by F0, F90, F180, F270, mF0, mF90, mF180, and mF270. The horizontal (vertical) dimension of the shear graph is corresponding with the horizontal dimension (vertical dimension) of the image plane of the reference picture. All six poses are displayed in each shear graph. Rot90, Rot180, and Rot 270 refer to the different comparisons with the reference picture.

As was mentioned in the discussion dealing with the effect of mirror reflection, one of the possible interpretations for the shears observed between the externalised percepts based on pictures differing by a particular transformation is that a 'mental viewpoint' has been located differently (see also Cornelis et al 2003). Koenderink et al (2001), being the first to propose this mental viewpoint interpretation, used (as one of their pictures) a depiction of the bilaterally symmetrical turtle of Brancusi in an oblique view. Conducting different tasks to externalise the percept (ie different crosssection reproduction tasks), they found that participants resolved the picture ambiguities in a specific way. Koenderink et al suggested that the location of the mental viewpoint was related to the 'generic' view of the turtle: it seemed to be situated above the axis of symmetry of the object. 

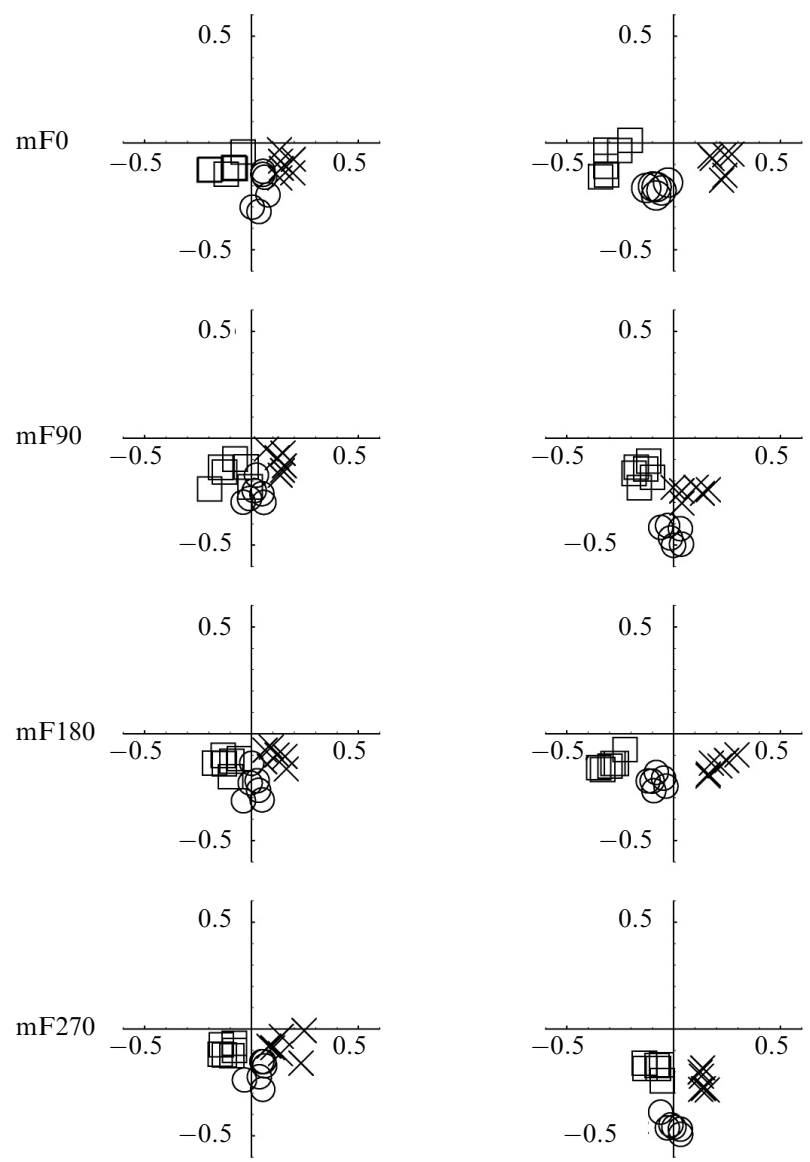

Figure 13 (continued)

In this respect it is also interesting to refer to a study performed by Mamassian and Landy (1998) in which participants were presented simple line drawings and were asked to decide, on the basis of their first impression, if they perceived a saddle-shaped or an egg-shaped surface. Modeling their data, Mamassian and Landy incorporated three assumptions, all of them necessary to provide a good fit for the data. One of the proposed assumptions was the bias for surface normals pointing upwards, interpreted as a preference to perceive the object as if one were looking at it from above. Moreover, the preference for saddle-shaped and egg-shaped surfaces was different depending on the orientation of the line drawing. When one of the line drawings was oriented so that the symmetry axis was vertical (or nearly horizontal), the line drawing was interpreted as egg-shaped (saddle-shaped). Thus, line drawings differing in orientation by $90^{\circ}$ apparently yielded different perceptual results.

Both of these findings, as well as those of the previous study, show that perceivers bring to bear all kinds of assumptions when interpreting flat pictures, both with respect to the generic nature of their viewpoint (Freeman 1994) as well as with respect to certain structural properties or regularities of the objects depicted in the pictures, such as object symmetry (van Lier and Wagemans 1999) or orthogonality of angles between parts (Vanrie et al 2001, 2002; Willems and Wagemans 2000). Photographers and artists appear to be well aware of these assumptions when they want to depict objects in so-called canonical orientations (Snodgrass and Vanderwart 1980; Verfaillie and Boutsen 1995; Wagemans et al 2008). 
Rotating the various pictures around $90^{\circ}$ or $270^{\circ}$ evidently resulted in a change of orientation of not only the frame format but also the intrinsic axes of the depicted object. The frame format and the intrinsic axes of the rotated picture versions were perpendicularly related to those of the reference pictures. In contrast, when rotating the pictures by $180^{\circ}$, the orientation of the frame format and the intrinsic axes of the depicted object obviously stayed the same. Therefore, one might have expected that the effects of rotation by $90^{\circ}$ or $270^{\circ}$ would be larger or different compared to the effects of a rotation around $180^{\circ}$. Furthermore, Mach asserted (1886/1959) that the 'affinity' of form is apparent between the reference figure and its $180^{\circ}$-rotated version, but much less so for the $90^{\circ}$-rotated version. In addition, Dearborn's results (1899; as cited in Rock 1973) suggested that the phenomenal similarity between the original figures and the $90^{\circ}$ - and the $270^{\circ}$-rotated versions was less obvious than the phenomenal similarity between the original figures and the $180^{\circ}$-versions. We, however, did not find support for the assertions/findings mentioned above: the pictorial relief differences were not smaller for the $180^{\circ}$ rotations than for the $90^{\circ}$ or $270^{\circ}$ rotations. Moreover, as we discussed above, the pictorial relief differences for the $180^{\circ}$ rotation as well as the $90^{\circ}$ and $270^{\circ}$ rotations could be largely ascribed to shears.

Part of the discrepancy between the viewpoint effects observed in our study and those observed in earlier studies may be rooted in the large stimulus and task differences (see also section 5). In particular, we have compared 3-D percepts obtained through a direct perceptual method applied to different views rather than asking observers to perform some matching or comparison task on the different views (giving no direct access to their 3-D percepts), as is usually done in studies focused on shape equivalence (eg Vanrie et al 2001; Wagemans 1992, 1993; Wagemans et al 1997, 2000; Willems and Wagemans 2001). Moreover, it has been noted before that the mental processes involved in shape perception may be different in contexts with side-by-side comparisons than in contexts in which single pictures must be related to internal representations (eg Wagemans et al 1996; Willems and Wagemans 2001).

\section{General conclusion}

We studied here the effect of two isomorphic transformations, more specifically mirror reflection and rotation, of a picture on the shape percept of the depicted object. Both kinds of transformations kept the intrinsic geometrical layout constant while the orientation of the picture changed: only the pixels were interchanged around an axis in the case of mirror reflection or around a point in the case of rotation. We investigated if mirror reflection and rotation of a picture affected the pictorial object-the percept of the object depicted in the picture.

The percept of a depicted 3-D object is not fully constrained by the content of the depiction. For instance, a 3-D object the depth values of which have been transformed affinely (eg scaled and sheared in depth) can still produce the same picture as the original 3-D object. Pictures are inherently ambiguous. Therefore, when looking into the picture, there are an infinite number of equally valid interpretations (percepts); the picture ambiguities can be solved in various ways.

In order to externalise the percept of the depicted object, we used a direct approach - the gauge-figure method. Participants were asked to perform a simple, visual task: adjusting a gauge figure so that it visually seemed to fit in the depicted scene. From these gauge-figure settings, pictorial reliefs were computed for all pictures. Subsequently, comparisons were performed between pictorial reliefs based on pictures that differed by the specific transformation investigated. Thus, we did not, as has often been the case in studies on perceptual shape equivalence, ask the participants to make some kind of global judgment of objects presented in various orientations. Furthermore, we focused more purely on the effect of the transformation itself. Therefore, we did not only perform 
comparisons between the pictorial reliefs based on the original photographs and their transformed versions, but included all possible comparisons between the pictorial reliefs based on pictures that differed by the particular transformation.

We have found that the transformation applied to the picture generally affected the perception of the depicted object. All transformations showed considerable differences between the pictorial reliefs of the reference pictures and the transformed versions, except for the mirror reflection around the vertical axis. The pictorial reliefs of the reference pictures and the pictorial reliefs of the left-right mirror-reflected versions were highly similar, providing additional support for the special status of vertical (left - right) symmetry compared to horizontal (up-down) symmetry and rotation. Furthermore, in all cases the effect of the transformation applied to the picture could be described in a well-specified way. The differences between the pictorial reliefs of the reference pictures and their transformed versions were affinely related. Different solutions to the depth ambiguities inherent in pictures were triggered, depending on the transformation applied onto the picture. Hence, the beholder's share in the interpretation of the depicted object was different for the different pictures.

Acknowledgments. We would like to thank Huib de Ridder and Kees Jorens for participation in the time-consuming experiments, and Huib de Ridder, Imre Horvath, René van Egmond, Arno Koning, and an anonymous reviewer for commenting on previous drafts of this article.

\section{References}

Barlow H B, Reeves B C, 1979 "The versatility and absolute efficiency of detecting mirror symmetry in random dot displays" Vision Research $19783-793$

Belhumeur P N, Kriegman D J, Yuille A L, 1999 "The bas-relief ambiguity" International Journal of Computer Vision 3533 - 34

Bornstein M H, Ferdinandsen K, Gross C G, 1981 "Perception of symmetry in infancy" Developmental Psychology $1782-86$

Bornstein M H, Krinsky S J, 1985 "Perception of symmetry in infancy: The salience of vertical symmetry and the perception of pattern wholes" Journal of Experimental Child Psychology $391-19$

Corballis M C, Roldan C E, 1975 "Detection of symmetry as a function of angular orientation" Journal of Experimental Psychology: Human Perception and Performance 1221 -230

Cornelis E V K, Doorn A J van, Ridder H de, 2003 "Mirror-reflecting pictures of an object: What happens to the shape percept?" Perception \& Psychophysics $651110-1125$

Cutting J E, 1988 "Affine distortions of pictorial space: some predictions for Goldstein (1987) that La Gournerie (1859) might have made" Journal of Experimental Psychology: Human Perception and Performance $14305-311$

Deręgowski J B, 1989 "Real space and represented space: Cross-cultural perspectives" Behavioral and Brain Sciences $1251-119$

Evans C E, Wenderoth P, Cheng K, 2000 "Detection of bilateral symmetry in complex biological images" Perception $2931-42$

Freeman W T, 1994 "The generic viewpoint assumption in a framework for visual perception" Nature $368542-545$

Gibson J J, 1954 "A theory of pictorial perception" Audio-Visual Communication Review 2 3-23

Gibson J J, 1979 The Ecological Approach to Visual Perception (Boston, MA: Houghton Mifflin)

Goldmeier E, 1936/1972 "Similarity in visually perceived forms" Psychological Issues 8 14-133

Gombrich E H, 1977 Art and Illusion 5th edition (Oxford: Phaidon Press)

Jolicoeur P, 1985 "The time to name disoriented natural objects" Memory \& Cognition 13 289-303

Julesz B, 1971 Foundations of Cyclopean Perception (Chicago, IL: University of Chicago Press)

Koenderink J J, Doorn A J van, 2003 "Pictorial space", in Looking into Pictures: An Interdisciplinary Approach to Pictorial Space Eds H.Hecht, R Schwartz, M Atherton (Cambridge, MA: MIT Press) pp $239-403$

Koenderink J J, Doorn A J van, Kappers A M L, 1992 "Surface perception in pictures" Perception Psychophysics $\mathbf{5 2} 487-496$

Koenderink J J, Doorn A J van, Kappers A M L, 1994 "On so-called paradoxical monocular stereoscopy" Perception $23583-594$

Koenderink J J, Doorn A J van, Kappers A M L, Todd J T, 2001 "Ambiguity and the 'mental eye' in pictorial relief" Perception $30431-448$ 
Kubovy M, 1986 The Psychology of Perspective and Renaissance Art (Cambridge: Cambridge University Press)

Kukkonen H T, Foster D H, Wood J R, Wagemans J, Van Gool L, 1996 "Qualitative cues in the discrimination of affine-transformed minimal patterns" Perception $25195-206$

Lawson R, 1999 "Achieving visual object constancy across plane rotation and depth rotation" Acta Psychologica $102221-245$

Lawson R, Jolicoeur P, 1998 "The effects of plane rotation on the recognition of brief masked pictures of familiar objects" Memory \& Cognition $26791-803$

Lier R van, Wagemans J, 1999 "From images to objects: Global and local completions of selfoccluded parts" Journal of Experimental Psychology: Human Perception and Performance $\mathbf{2 5}$ $1721-1741$

Mach E, 1886/1959 The Analysis of Sensations and the Relation of the Physical to the Psychical translated by C M Williams (New York: Dover)

Mamassian P, Landy M S, 1998 "Observer biases in the 3D interpretation of line drawings" Vision Research $382817-2832$

Moeller A P, Thornhill R, 1998 "Bilateral symmetry and sexual selection: A meta-analysis" American Naturalist 151(2) $174-192$

Palmer S E, Hemenway K, 1978 "Orientation and symmetry: Effects of multiple, rotational, and near symmetries" Journal of Experimental Psychology: Human Perception and Performance 4 $691-702$

Perrett D I, Burt D M, Penton-Voak I S, Lee K J, Rowland D A, Edwards R, 1999 "Symmetry and human facial attractiveness" Evolution and Human Behavior 20 295-307

Pirenne M H, 1970 Optics, Painting and Photography (Cambridge: Cambridge University Press)

Rhodes G, Proffitt F, Grady J M, Sumich A, 1998 "Facial symmetry and the perception of beauty" Psychonomic Bulletin \& Review $5659-669$

Rock I, 1973 Orientation and Form (New York: Academic Press)

Royer F L, 1981 "Detection of symmetry" Journal of Experimental Psychology: Human Perception and Performance $71186-1210$

Shepard R N, Cooper L A, 1982 Mental Images and Their Transformations (Cambridge, MA: MIT Press

Shepard R N, Metzler J, 1971 "Mental rotation of three-dimensional objects" Science 171701 - 703

Snodgrass J G, Vanderwart M, 1980 "A standardized set of 260 pictures: Norms for name agreement, image agreement, familiarity, and visual complexity" Journal of Experimental Psychology: Human Learning and Memory $6174-215$

Van Gool L, Moons T, Pauwels E, Wagemans J, 1994 "Invariance from the Euclidean geometer's perspective" Perception $23547-561$

Vanrie J, Béatse E, Wagemans J, Sunaert S, Van Hecke P, 2002 "Mental rotation versus invariant features in object perception from different viewpoints: An fMRI study" Neuropsychologia $40917-930$

Vanrie J, Willems B, Wagemans J, 2001 "Multiple routes to object matching from different viewpoints: Mental rotation versus invariant features" Perception $301047-1056$

Verfaillie K, Boutsen L, 1995 "A corpus of 714 full-color images of depth-rotated objects" Perception \& Psychophysics $57925-961$

Wagemans J, 1992 "Perceptual use of nonaccidental properties" Canadian Journal of Psychology $46236-279$

Wagemans J, 1993 "Skewed symmetry: A nonaccidental property used to perceive visual forms" Journal of Experimental Psychology: Human Perception and Performance 19 364-380

Wagemans J, 1995 "Detection of visual symmetries" Spatial Vision 9 9-32

Wagemans J, De Winter J, Op de Beeck H P, Ploeger A, Beckers T, Vanroose P, 2008 "Identification of everyday objects on the basis of silhouette and outline versions" Perception 37 207-244

Wagemans J, Lamote C, Van Gool L, 1997 "Shape equivalence under perspective and projective transformations" Psychonomic Bulletin \& Review 4 248-253

Wagemans J, Van Gool L, d'Ydewalle G, 1992 "Orientational effects and component processes in symmetry detection" The Quarterly Journal of Experimental Psychology 44A 475-508

Wagemans J, Van Gool L, Lamote C, 1996 "The visual system's measurement of invariants need not itself be invariant" Psychological Science $7232-236$

Wagemans J, Van Gool L, Lamote C, Foster D H, 2000 "Minimal information to determine affine shape equivalence" Journal of Experimental Psychology: Human Perception and Performance 26 $443-468$

Wenderoth P, 1994 “The salience of vertical symmetry” Perception 23221 -236 
Wenderoth P, 1996 "The effects of dot pattern parameters and constraints on the relative salience of vertical bilateral symmetry" Vision Research $362311-2320$

Willems B, Wagemans J, 2000 "The viewpoint-dependency of veridicality: Psychophysics and modelling" Vision Research $403017-3027$

Willems B, Wagemans J, 2001 "Matching multi-component objects from different viewpoints: Mental rotation as normalization?" Journal of Experimental Psychology: Human Perception and Performance 27 1090-1115

Zimmer A, 1984 "Foundations for the measurement of phenomenal symmetry" Gestalt Theory 6 $118-157$ 


\section{PERTEPTION}

VOLUME 382009

www.perceptionweb.com

Conditions of use. This article may be downloaded from the Perception website for personal research by members of subscribing organisations. Authors are entitled to distribute their own article (in printed form or by e-mail) to up to 50 people. This PDF may not be placed on any website (or other online distribution system) without permission of the publisher. 